\title{
Treatment strategies for coronary in-stent restenosis: systematic review and hierarchical Bayesian network meta-analysis of 24 randomised trials and 4880 patients
}

\author{
Daniele Giacoppo, ${ }^{1}$ Giuseppe Gargiulo, ${ }^{1}$ Patrizia Aruta, ${ }^{1}$ Piera Capranzano, ${ }^{1,2}$ Corrado Tamburino, ${ }^{1,2}$ \\ Davide Capodanno ${ }^{1,2}$
}

${ }^{1}$ Department, Ferrarotto

Hospital, Catania, Italy

${ }^{2}$ Department of General Surgery and Medical Surgical

Specialties, Ferrarotto Hospital,

University of Catania, 95124

Catania, Italy

Correspondence to:

D Capodanno

dcapodanno@gmail.com

Additional material is published online only. To view please visit the journal online (http://dx.doi. org/10.1136/bmj.h5392)

Cite this as: $B M J$ 2015;351:h5392 doi: 10.1136/bmj.h5392

Accepted: 22 September 2015

\section{ABSTRACT}

\section{STUDY QUESTION}

What is the most safe and effective interventional treatment for coronary in-stent restenosis?

METHODS

In a hierarchical Bayesian network meta-analysis, PubMed, Embase, Scopus, Cochrane Library, Web of Science, ScienceDirect, and major scientific websites were screened up to 10 August 2015. Randomised controlled trials of patients with any type of coronary in-stent restenosis (either of bare metal stents or drug eluting stents; and either first or recurrent instances) were included. Trials including multiple treatments at the same time in the same group or comparing variants of the same intervention were excluded. Primary endpoints were target lesion revascularisation and late lumen loss, both at six to 12 months. The main analysis was complemented by network subanalyses, standard pairwise comparisons, and subgroup and sensitivity analyses.

\section{STUDY ANSWER AND LIMITATIONS}

Twenty four trials (4880 patients), including seven interventional treatments, were identified. Compared with plain balloons, bare metal stents, brachytherapy, rotational atherectomy, and cutting balloons, drug coated balloons and drug eluting stents were associated with a reduced risk of target lesion revascularisation and major adverse cardiac events, and with reduced late lumen loss. Treatment ranking indicated that drug eluting stents had the highest

\section{WHAT IS ALREADY KNOWN ON THIS TOPIC}

Management of patients with coronary in-stent restenosis is difficult, owing to many factors such as varying causes (aggressive neointimal proliferation, neoatherosclerosis) and the high tendency to recur

In the past 20 years, several strategies have been proposed to counteract in-stent restenosis, but randomised trials comparing different treatments have given mixed and inconclusive results

\section{WHAT THIS STUDY ADDS}

In a network meta-analysis, contemporary treatment strategies for coronary in-stent restenosis (drug coated balloons and drug eluting stents) were compared with other treatments investigated over the years

Pooled evidence suggested comparable clinical and angiographic antirestenotic efficacy for drug coated balloons and drug eluting stents; plain balloons, bare metal stents, brachytherapy, rotational atherectomy, and cutting balloons were associated with an increased risk of target lesion revascularisation and inferior angiographic results

No differences in death, myocardial infarction, and stent thrombosis were noted across all the treatments investigated

probability (61.4\%) of being the most effective for target lesion vascularisation; drug coated balloons were similarly indicated as the most effective treatment for late lumen loss (probability 70.3\%). The comparative efficacy of drug coated balloons and drug eluting stents was similar for target lesion revascularisation (summary odds ratio 1.10, 95\% credible interval 0.59 to 2.01 ) and late lumen loss reduction (mean difference in minimum lumen diameter $0.04 \mathrm{~mm}, 95 \%$ credible interval -0.20 to $0.10)$. Risks of death, myocardial infarction, and stent thrombosis were comparable across all treatments, but these analyses were limited by a low number of events. Trials had heterogeneity regarding investigation periods, baseline characteristics, and endpoint reporting, with a lack of information at long term follow-up. Direct and indirect evidence was also inconsistent for the comparison between drug eluting stents and drug coated balloons.

\section{WHAT THIS STUDY ADDS}

Compared with other currently available interventional treatments for coronary in-stent restenosis, drug coated balloons and drug eluting stents are associated with superior clinical and angiographic outcomes, with a similar comparative efficacy.

FUNDING, COMPETING INTERESTS, DATA SHARING This study received no external funding. The authors declare no competing interests. No additional data available.

\section{Introduction}

Drug eluting stents have substantially reduced the risk of coronary in-stent restenosis and the need for target lesion revascularisation compared with bare metal stents by counteracting the exuberant neointimal proliferation that follows stent implantation. ${ }^{1}$ However, current rates of in-stent restenosis in clinical practice remain higher than $10 \% .^{23}$

Management of patients with in-stent restenosis is challenging and the best therapeutic strategy remains unclear. ${ }^{4}$ Treatment with plain balloons is technically simple and generally associated with acceptable procedural success, due to axial and longitudinal tissue extrusion and incremental stent expansion. ${ }^{5}$ However, observational studies and randomised trials have consistently shown inferior clinical and angiographic results compared with implantation of a second drug eluting stent. ${ }^{6-8}$ Nevertheless, plain balloon angioplasty is still used for in-stent restenosis treatment in a consistent proportion of patients. ${ }^{910}$ Furthermore, in-stent implantation of drug eluting stents tends to be 
restricted to a limited proportion of patients, owing to concerns related to positioning a permanent additional stent layer. Additional layers promote further endothelial growth as well as potential mechanical complications, either acutely or later on (such as fracture, malapposition, thrombosis). 411

Recently, drug coated balloons have emerged as promising alternatives to drug eluting stents for in-stent restenosis, but large randomised trials comparing drug coated balloons with other therapeutic options are limited. .14 $^{14}$ Other treatment options for in-stent restenosis have been used over time with heterogeneous results, including implantation of bare metal stents, vascular brachytherapy, rotational atherectomy, and cutting balloons. ${ }^{415}$

Network meta-analyses are an extension of traditional pairwise meta-analyses that enable the simultaneous pooling of data from clinical trials comparing at least two treatments and strengthen the inference on the relative efficacy of each treatment by including both direct and indirect information. ${ }^{17}$ The objective of this systematic review and hierarchical Bayesian network meta-analysis was to pool data from randomised trials comparing at least two interventional treatments for coronary in-stent restenosis and to identify which strategy is eventually the most effective and safe.

\section{Methods}

\section{Data sources and study strategy}

This meta-analysis was performed in agreement with the PRISMA (preferred reporting items for systematic reviews and meta-analyses) statement, the PRISMA network meta-analysis extension statement, and the Cochrane Collaboration recommendations (web appendix, PRISMA checklist). ${ }^{18-20}$ Randomised trials comparing at least two different treatments were searched in PubMed, Embase, Scopus, Cochrane Library, Web of Science, and ScienceDirect electronic databases, as well as major scientific websites (www.tctmd.com, www.pcronline.com, www.clinicaltrials.gov, www.clinicaltrialresults.org, www.acc.org, www.heart.org, www.medscape.com). Abstracts and presentations from major cardiovascular meetings were considered. The electronic search process was integrated by tangential exploration of bibliography of relevant reviews on in-stent restenosis and major interventional cardiology books.

The web appendix reports the combination of subject headings used for studies identification. No language restriction or filters were imposed. The search was performed from the date of databases' inception to 10 August 2015.

\section{Selection criteria and study design}

Inclusion criteria were the following: randomised controlled trials of patients with coronary in-stent restenosis; patients of any age, sex, ischaemic risk profile, and clinical presentation; either in-stent restenosis of a previously implanted bare metal stent (BMS-ISR) or in-stent restenosis of a previously implanted drug eluting stent (DES-ISR); and either first or recurrent instances of in-stent restenosis. Exclusion criteria were: non-interventional treatment for in-stent restenosis; comparison between variants of the same type of device (same treatment group); and investigations including combinations of multiple treatments in the same group at the same time, except for the use of plain balloons or cutting balloons for lesion preparation before other treatments (that is, brachytherapy or stent implantation).

Coronary in-stent restenosis is classically defined as the angiographic detection of a recurrent stenosis with diameter greater than $50 \%$ at the stent segment or its $5 \mathrm{~mm}$ adjacent segments (in-segment restenosis). However, some trials have considered only the segment of the implanted stent, without inclusion of proximal and distal edges (in-lesion restenosis). ${ }^{21}$ In this meta-analysis, we prioritised events related to in-segment restenosis; if they were not available, we included events related to in-lesion restenosisrelated events.

The primary clinical endpoint was target lesion revascularisation at six to 12 months, defined as any repeated revascularisation involving the target lesion, both percutaneous and surgical; if target lesion revascularisation was not available, target vessel revascularisation was pooled. ${ }^{22}$ The primary angiographic endpoint was late lumen loss at six to 12 months, defined as the difference between the minimum lumen diameter after the procedure and at follow-up, as evaluated by quantitative coronary angiography. ${ }^{23}$ Late lumen loss was designated as a coprimary endpoint, because this angiographic measure is known to be consistent and reliable in discriminating the propensity for restenosis. $^{24}$

Secondary clinical endpoints were death, myocardial infarction, stent thrombosis, and combined major adverse cardiac events including death, myocardial infarction, target lesion revascularisation, and stent thrombosis. ${ }^{22}$ The definition of major adverse cardiac events was modified retrospectively because of the observed heterogeneity across the trials, allowing for the inclusion of target vessel revascularisation instead of target lesion revascularisation. Very dissimilar definitions, however, were not allowed, leading to the exclusion of the corresponding trial from the meta-analysis for major adverse cardiac events. Secondary angiographic endpoints were minimum lumen diameter and binary restenosis at six to 12 months. ${ }^{23}$

Search and screening of retrieved records at the title and abstract level were independently performed by three reviewers (DG, PA, GG). The same three reviewers assessed full text eligibility of the identified trials and discrepancies were resolved by consensus under the supervision of other two investigators (PC, DC). The validity of the meta-analysis was assessed by qualitative appraisal of study designs and methods before statistical analyses were performed, with the use of the risk of bias tool recommended by the Cochrane Collaboration. ${ }^{20}$ Data from original reports were collected into specific electronic spreadsheets. 


\section{Patient involvement}

No patients were involved in setting the research question or the outcome measures, nor were they involved in the design and implementation of the study. There are no plans to involve patients in dissemination.

\section{Statistical analyses}

Data used in this meta-analysis were intention to treat. Most of the included trials did not report as treated results. A hierarchical Bayesian network meta-analysis was carried out for each endpoint using random effects consistency models. ${ }^{25-27}$ Briefly, in a network meta-analysis, each study provides an estimate of the study specific treatment effects, which are assumed to be similar and exchangeable (that is, transitivity), deriving from a normal common distribution. ${ }^{28}$ Each relative treatment effect estimate results from the combination of the direct evidence between the two treatments and the indirect evidence deriving from the network meta-analysis, which are assumed to be coherent. ${ }^{2629}$ When a direct connection between two treatments is not available, the effect estimates derives only from indirect evidence. ${ }^{26} 29$

We used random effects models because they are probably the most appropriate and conservative methodology to account for between-trial heterogeneity within each comparison. ${ }^{29} 30$ Models were computed with Markov chain Monte Carlo simulations, using three chains with over-dispersed initial values, with Gibbs sampling based on 100000 iterations after a burn-in phase of 50000 iterations. Non-informative or vague priors for the overall mean effect $\left(\theta \sim \mathrm{N}\left(0,100^{2}\right)\right)$ and the between-study standard deviation ( $\tau \sim$ uniform $(0,2))$ were given. ${ }^{272931}$ We evaluated convergence according to Brooks-Gelman-Rubin. ${ }^{32}$

The information was imputed according to the arm based approach, and modelled by use of binomial data (binomial likelihood, logit link) or sample means (normal likelihood, identity link) with normal distribution, according to the specific type of outcome explored. ${ }^{1727}$ We computed posterior mean effect, odds ratio or mean difference, where appropriate, and 95\% credible intervals for each comparison. The included treatments were ranked to define the probability associated to each one being the best interventional strategy when significant variations in treatment effect were observed. ${ }^{30} 33$

We assessed inconsistency by comparing statistics for the deviance information criterion in fitted consistency and inconsistency models, and by contrasting direct evidence with indirect evidence from the entire network on each node (node-split). ${ }^{25-27}$ A Bayesian $P$ value was calculated to estimate the measure of the conflict between direct and indirect evidence by counting the proportion of times the direct treatment effect exceeded the indirect treatment effect. ${ }^{26}$ The estimates of Bayesian pairwise comparisons were also calculated with results complemented by standard frequentist DerSimonian-Laird meta-analyses with inverse variance weighting. ${ }^{34}$ In the frequentist framework, the pooled estimates were quantified as summary odds ratios or mean differences, where appropriate, and corresponding 95\% confidence intervals.

The amount of the observed variance reflecting real differences in the effect size across the included trials was graded with the $\mathrm{Q}$ test and $\mathrm{I}^{2}$ statistic with values representing mild, moderate, and severe heterogeneity ( $<25 \%, 25-75 \%$, and $>75 \%$, respectively). ${ }^{35}$ The variance of the true effect size across the included trials $\left(\tau^{2}\right)$ was calculated. ${ }^{36}$ We assessed publication bias and small study effect by visual inspection of comparison adjusted and contours enhanced funnel plots complemented by Peters' and Egger's tests, where appropriate. ${ }^{36-40}$

We did subgroup analyses according to the type of restenotic stent (BMS-ISR or DES-ISR), and the generation of drug eluting stent implanted for in-stent restenosis treatment (first or second generation). As another sensitivity analysis, we removed each trial from the others when results suggested the mean effect to be potentially driven by individual studies. ${ }^{36}$ All analyses were performed using $\mathrm{R}$ (version 3.1.1), Stata (version 12.1), and RevMan (version 5.3).

\section{Results}

Systematic review and qualitative assessment

A total of 24 trials $(n=4880)$ and seven interventional treatments (plain balloon, drug coated balloon, drug eluting stent, bare metal stent, brachytherapy, rotational atherectomy, and cutting balloon) were included. Fig 1 shows each phase of the screening process, and fig 2 shows the weighted network. The web appendix includes the list of acronyms and identification numbers (www.clinicaltrials.gov) of the trials included in the analysis. Potential sources of bias in trial design and investigational methods graded according to the

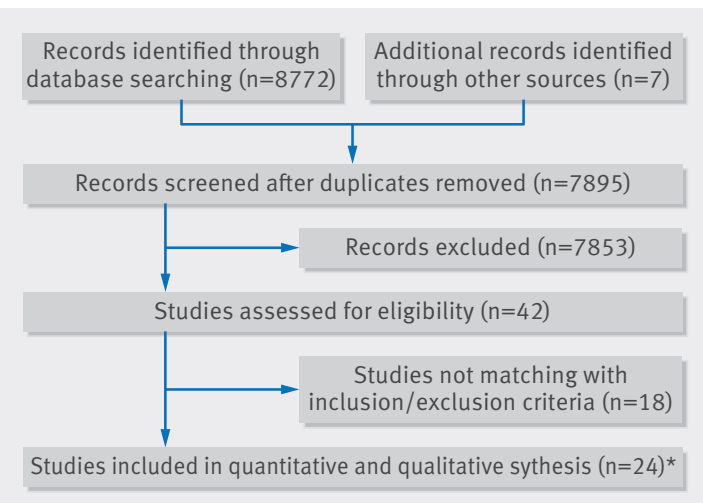

Fig 1 | Systematic search and screening process of trials. *The study by Ragosta and colleagues had two cohorts with independent randomisation processes that were separately included in the meta-analysis. Similarly, the study by Song and colleagues had two cohorts with independent randomisation processes, but only the first (cutting balloon $v$ sirolimus eluting stent) was included in this meta-analysis because the second (sirolimus eluting stent $v$ everolimus eluting stent) compared two variants of the same treatment. Finally, we considered the PACCOCATH ISR I and II trials together because the second study is the cohorts' extension of the first one 


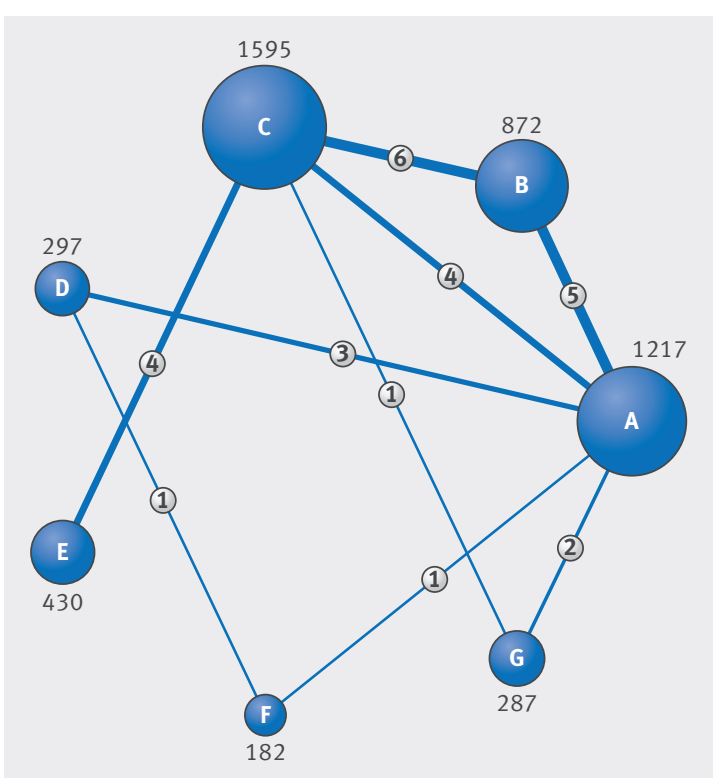

Fig 2 Network of interventional treatments included in meta-analysis. $A=$ plain balloon; $B=d r u g$ coated balloon; $C=$ drug eluting stent; $D=$ bare metal stent; $\mathrm{E}=$ brachytherapy; $\mathrm{F}=$ rotational atherectomy; $\mathrm{G}=$ cutting balloon. Numbers on connecting lines between each intervention=head to head comparisons; numbers next to specific interventions=patients receiving a treatment

Cochrane's Collaboration risk of bias tool (web fig 1) suggested most of the trials to be open label and therefore potentially affected by performance bias. ${ }^{20}$

Table 1 describes key characteristics of the included studies (design, samples size, treatments, in-stent restenosis definition, follow-up length, and original endpoints). Almost all trials were powered for angiographic endpoints, mainly late lumen loss, and scheduled for angiographic surveillance. Two trials did not plan midterm angiographic follow-up, and were excluded from all meta-analyses of angiographic endpoints. In about $70 \%$ of trials, angiographic follow-up was performed starting from six months after procedure, while 62.5\% of trials had planned clinical follow-up at 12 months. Almost $60 \%$ of trials included only patients with BMSISR, nearly $30 \%$ included only patients with DES-ISR, while two trials included both stent types of in-stent restenosis.

Table 2 summarises the clinical and angiographic characteristics of patients enrolled in each trial. The included participants had a mean age of 64 years, were prevalently male (76\%), and underwent repeated percutaneous coronary intervention mainly for silent ischaemia/stable angina or unstable angina. Prevalence of diabetes was highly variable across trials (14-62\%). Web table 1 reports all the inclusion and exclusion criteria of each trial.

\section{Bayesian network meta-analyses}

With respect to the primary clinical endpoint (fig 3), use of drug coated balloons or drug eluting stents markedly reduced the risk of target lesion revascularisation compared with all the other treatments. When com- pared directly, drug coated balloons and drug eluting stents had similar antirestenotic efficacy (summary odds ratio $1.10,95 \%$ credible interval 0.59 to 2.01 ). Treatment ranking reflected the consistent reduction in the risk of target lesion revascularisation associated with drug coated balloons or drug eluting stents over the other strategies, but also indicated that drug eluting stents had a higher probability (61.4\%) of being the best therapy. Rotational atherectomy was associated with the highest risk of target lesion revascularisation compared with the other treatments.

With respect to the primary angiographic endpoint (fig 4), use of a drug coated balloon or drug eluting stent were the most effective treatments, while use of a bare metal stent showed the highest mean difference in late lumen loss, followed by rotational atherectomy. Drug coated balloons emerged as the best therapy in treatment ranking (probability of $70.3 \%$ ), but the extent of the late lumen loss reduction compared with drug eluting stents was marginal (mean difference $-0.04 \mathrm{~mm}$, $95 \%$ credible interval -0.20 to 0.10 ).

Risk of major adverse cardiac events was consistently reduced with use of drug coated balloons or drug eluting stents, compared with all the other treatments (fig 5). Rotational atherectomy therapy led to an increased risk also when compared with poorly effective treatments such as brachytherapy and bare metal stent. The endpoint of major adverse cardiac events was mainly driven by target lesion revascularisation, since no remarkable differences across treatment strategies were noted in terms of death and myocardial infarction (fig 6). However, the incidences of death and myocardial infarction were overall extremely low and conclusions about these endpoints remain limited, especially for treatment comparisons supported by single or few trials. The angiographic superiority of drug coated balloons or drug eluting stents over the other treatments was also confirmed by evaluation of the angiographic secondary endpoints of minimum lumen diameter and binary restenosis (web fig 2). In particular, patients treated with a drug coated balloon or drug eluting stent achieved a higher minimum lumen diameter at follow-up than did the other treatment strategies, and the risk of binary restenosis generally followed the distribution observed for target lesion revascularisation.

All models converged adequately. Heterogeneity (global $\mathrm{I}^{2}$ ) was moderate for target lesion revascularisation (43.5\%), high for late lumen loss (95.3\%), and low to moderate for secondary endpoints (major adverse cardiac events $=8.5 \%$, death $=0 \%$, myocardial infarction $=11.8 \%$, minimum lumen diameter $=45.4 \%$, binary restenosis $=59.4 \%$ ). Model fitting was compared by use of the deviance information criterion and shown to be similar. The node-split in the analyses for target lesion revascularisation and late lumen loss showed a significant inconsistency in the comparison of drug coated balloons versus drug eluting stents. However, the nodesplit for the secondary endpoint analyses showed significant inconsistency between drug coated balloons and drug eluting stents only for myocardial infarction. 


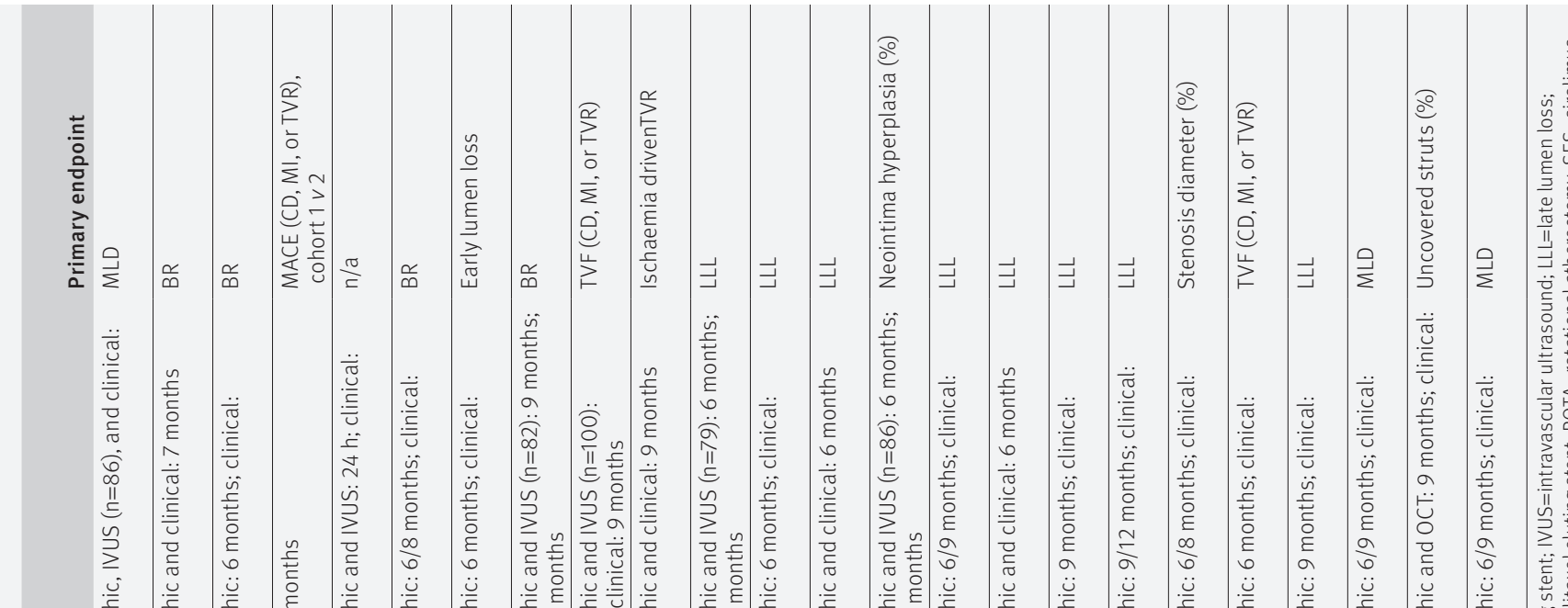

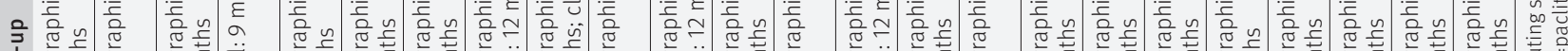

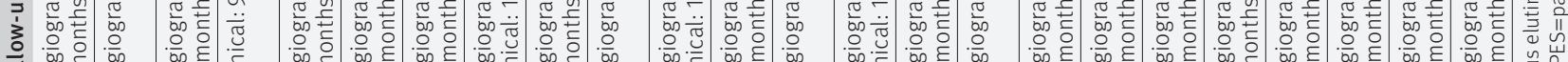

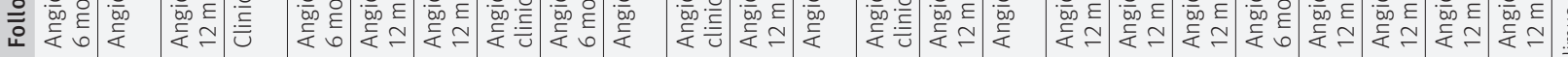

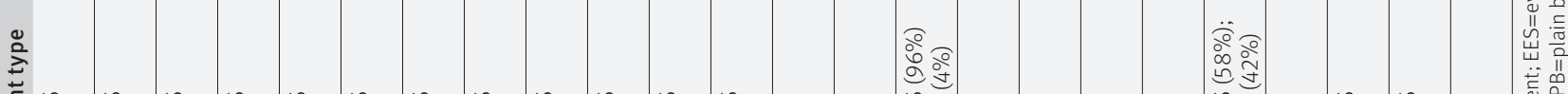

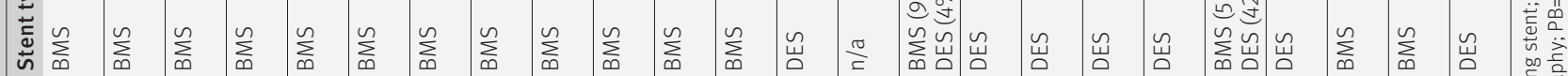

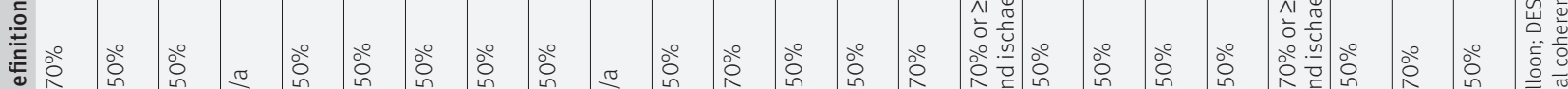

亭

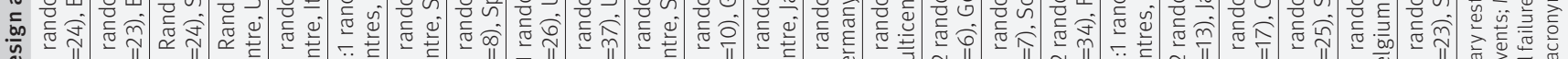

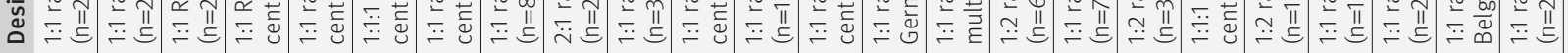


I

I!

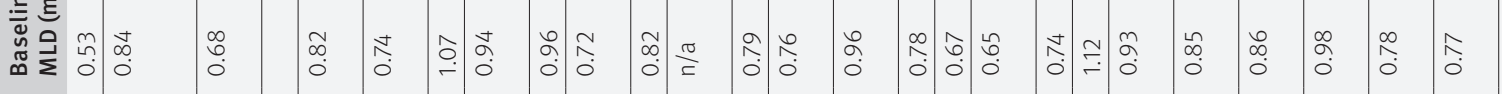

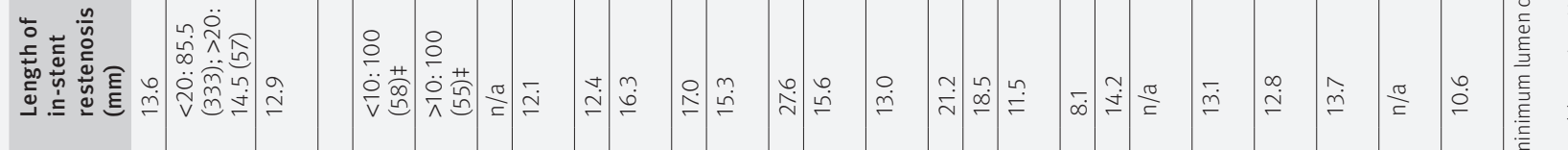

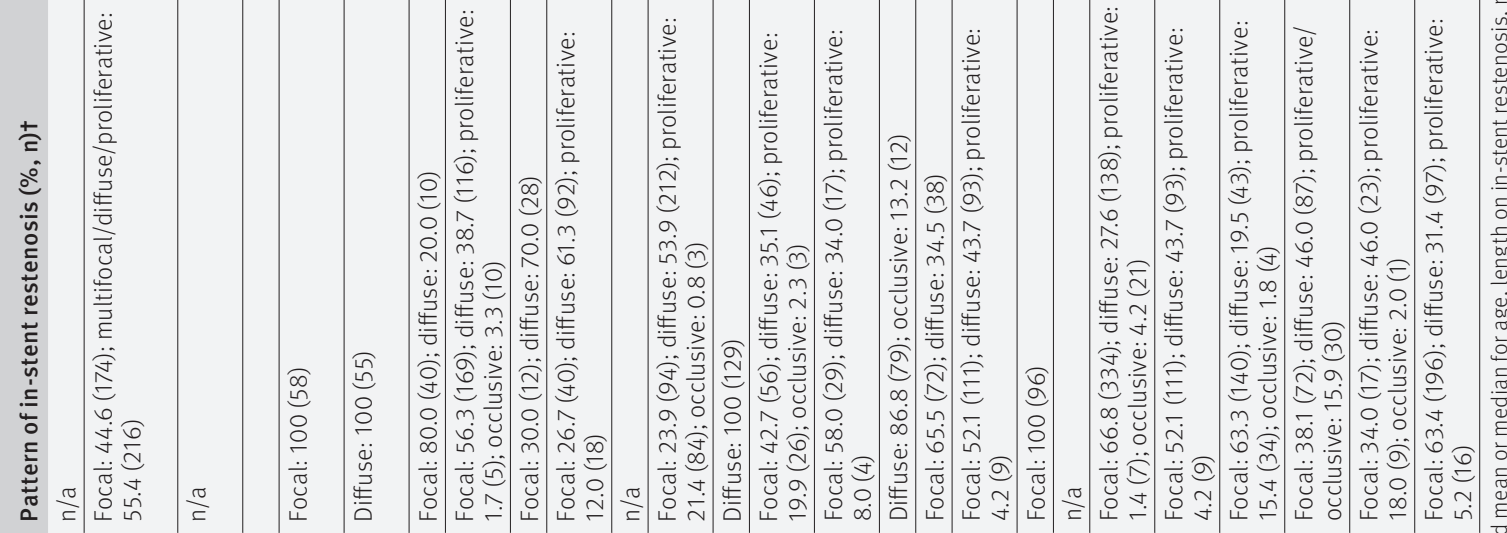

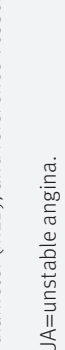

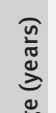

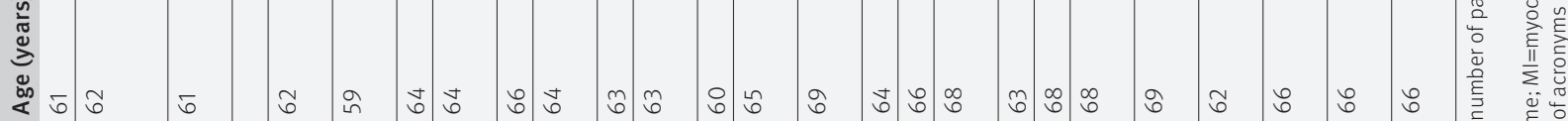




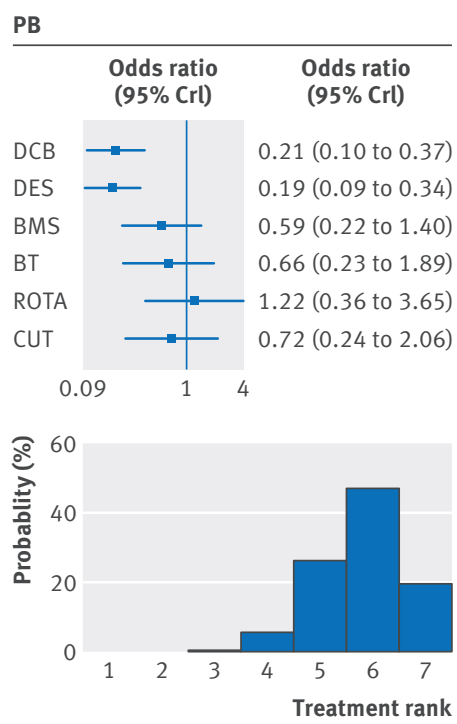

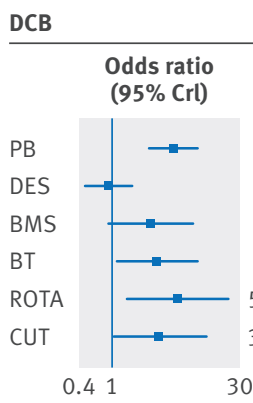
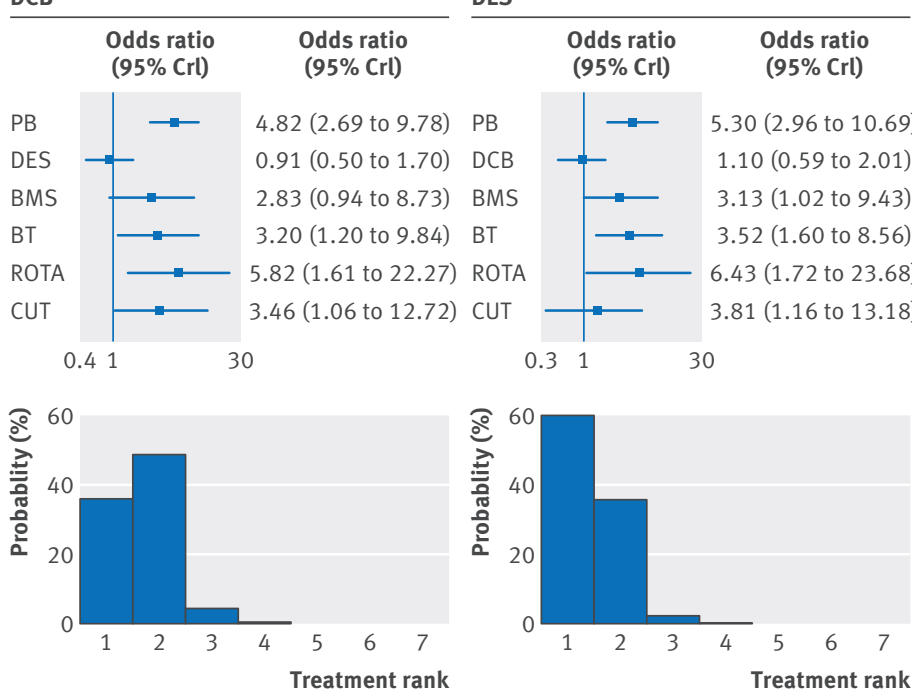

0.31

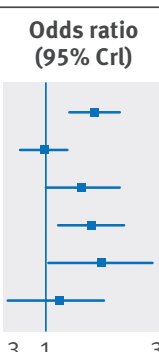

Odds ratio

(95\% Crl)
BMS
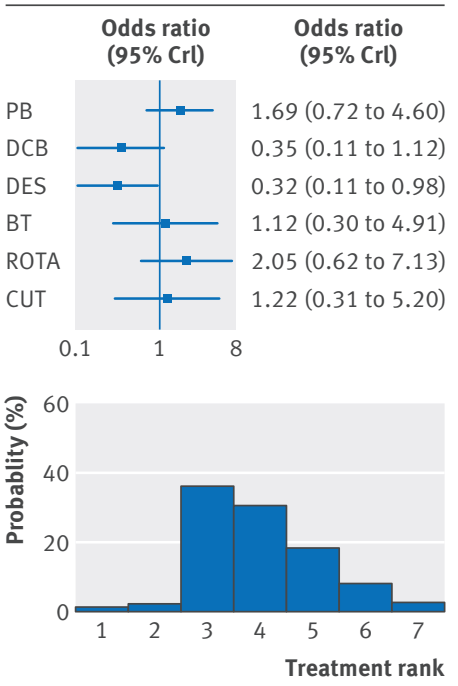

CUT

ROTA

Odds ratio

(95\% Crl)

\begin{tabular}{cc}
\hline Odds ratio & Odds ratio \\
$(95 \% \mathrm{Crl})$ & $(95 \% \mathrm{Crl})$
\end{tabular}

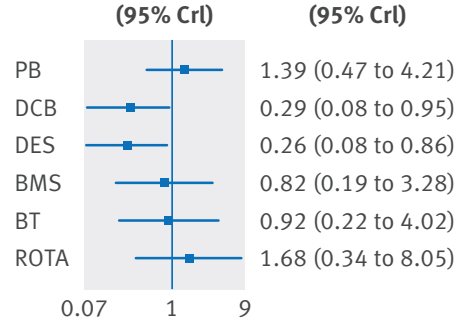

$0.82(0.27$ to 2.80$) \quad P B$ 0.17 (0.04 to 0.62$) \quad D C B$ 0.16 (0.04 to 0.58$)$ DES 0.49 ( 0.14 to 1.63$)$ BMS 0.55 (0.12 to 2.73) BT 0.59 (0.12 to 2.96) ROTA
We detected a significant inconsistency between comparisons involving cutting balloons for each endpoint.

\section{Bayesian network subanalyses and frequentist head} to head comparisons

The main network meta-analysis indicated that drug coated balloons and drug eluting stents were the most effective treatments; thus, we further investigated variations in mean effect, heterogeneity, and consistency in subanalyses. We did a network meta-analysis of closed loop plain balloons, drug coated balloons, and drug eluting stents (13 trials; 2417 patients) to reduce the inconsistency arising from indirect evidence. Web tables 2 and 3 show additional information on the trials included in this network meta-analysis. We also did standard, frequentist, pairwise meta-analyses to complement the results of the network meta-analysis.
Compared with plain balloons, use of drug coated balloons and drug eluting stents continued to be associated with a strong reduction in the risk of target lesion revascularisation (drug coated balloons $v$ plain balloons: summary odds ratio $0.21,95 \%$ credible interval 0.09 to 0.43 ; drug eluting stents $v$ plain balloons: 0.19 , 0.08 to 0.42 ; fig 7 ). Similar results were observed for the mean difference in late lumen loss $(-0.44 \mathrm{~mm},-0.63$ to $-0.27 ;-0.39 \mathrm{~mm},-0.60$ to -0.18 ; fig 8 ). After comparison of drug coated balloons with and drug eluting stents, the risk estimates of target lesion revascularisation and late lumen loss did not show a significant benefit favouring one treatment over the other. The node-split analysis showed that the pooled risk of target lesion revascularisation and late lumen loss between the two treatments in the network meta-analysis was differentially driven by indirect (plain balloon $v$ drug coated 
PB
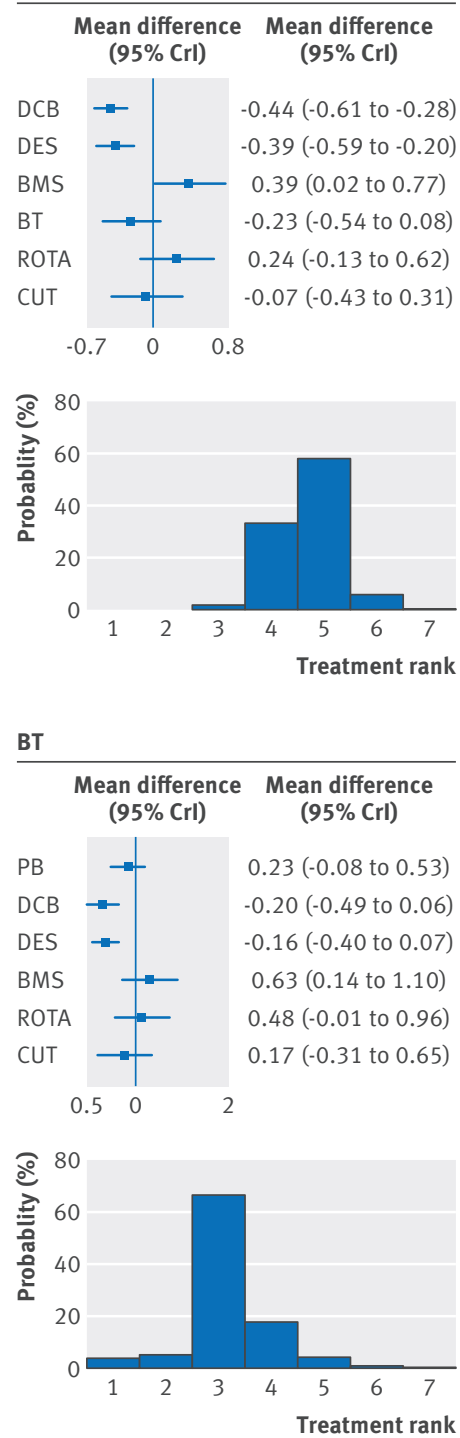

DCB
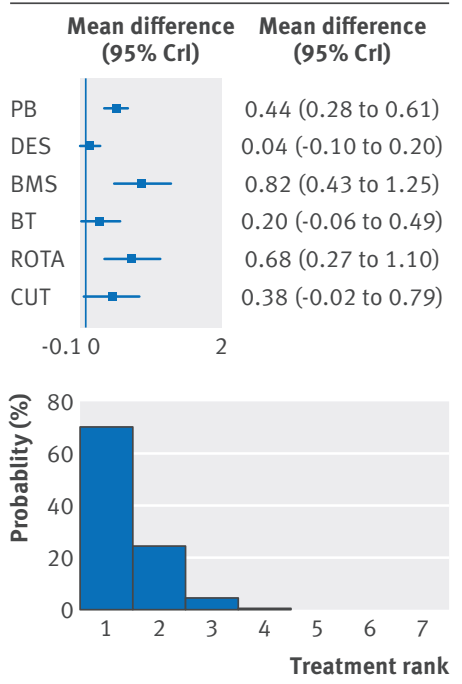

ROTA
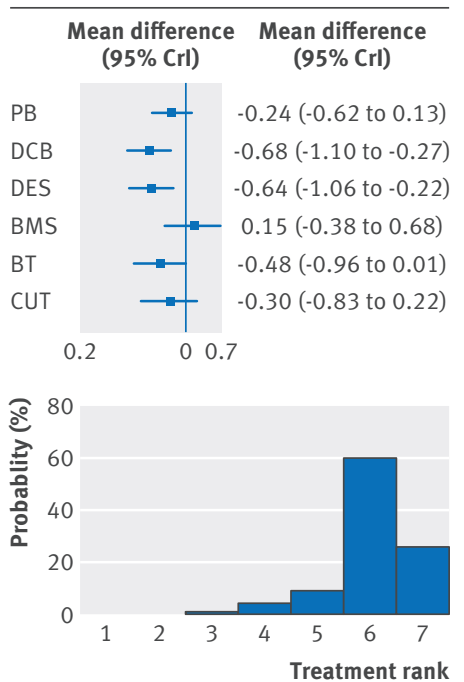

DES
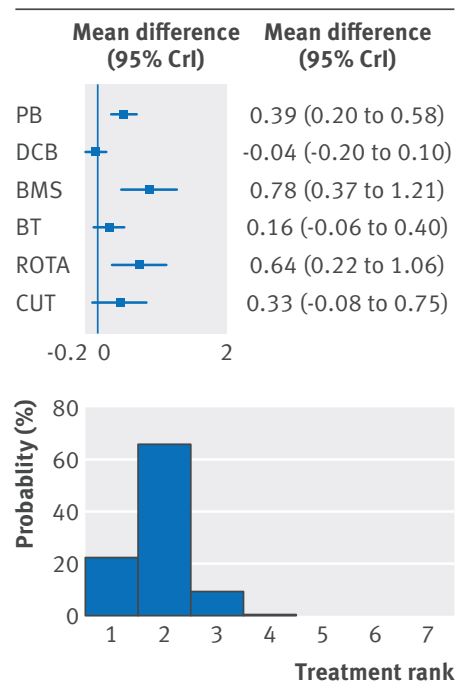

CUT
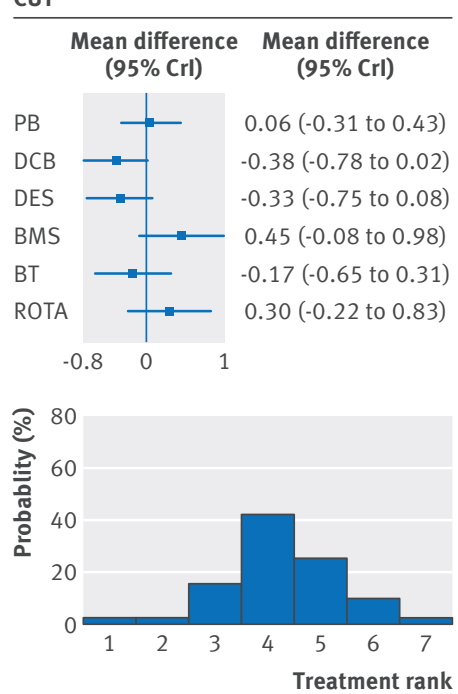

BMS
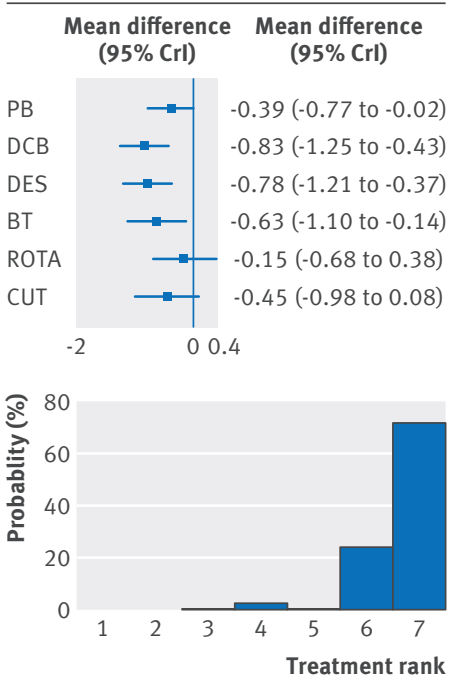

Fig 4 | Effect of interventional treatments on late lumen loss. Forest plots show relative effect of each treatment on late lumen loss as compared with a common reference treatment. Histograms are shown for each treatment reflecting corresponding probabilities for each position in the ranking of the seven strategies (rankograms). $I^{2}$ value=95.3\%. $P B=$ plain balloon; $D C B=$ drug coated balloon; $D E S=d r u g$ eluting stent; $B M S=b a r e$ metal stent; $\mathrm{BT}=$ brachytherapy; $\mathrm{ROTA}=$ rotational atherectomy; $\mathrm{CUT}=$ cutting balloon; $\mathrm{MD}=$ mean difference; $\mathrm{Crl}=\mathrm{credible}$ interval

balloon; and plain balloon $v$ drug eluting stent) and direct evidence (Bayesian values $\mathrm{P}=0.043$ for target lesion revascularisation and $\mathrm{P}=0.036$ for late lumen loss). Results of corresponding standard pairwise meta-analyses were concordant with those of the network meta-analysis.

At treatment ranking, use of plain balloons was associated with a $99.9 \%$ probability of being the least effective treatment both in terms of target lesion revascularisation and late lumen loss. The risk of major adverse cardiac events was reduced in patients treated with drug coated balloons and drug eluting stents compared with those treated with plain balloons, but similar effects were noted between the two strategies (web fig 3). The risk of death and myocardial infarction in both the network meta-analysis and standard pairwise meta-analyses tended to be lower with drug coated balloons than with drug eluting stents (web figs 4 and 5). But owing to the low number of events, this distribution could reflect the effect of chance.

\section{Subgroup and sensitivity analyses}

To explore whether earlier devices are affected by a differential response when treated for in-stent restenosis, we stratified patients by categories of BMS-ISR or DESISR, and re-evaluated the antirestenotic efficacy of plain balloons, drug coated balloons, and drug eluting stents (figs 9 and 10). Drug coated balloons and drug eluting stents were consistently associated with a significant reduction in the risk of target lesion revascularisation compared with plain balloons both in BMS-ISR and DES-ISR. Both the network and standard pairwise meta-analyses suggested that the magnitude of the benefit of drug coated balloons compared with plain 

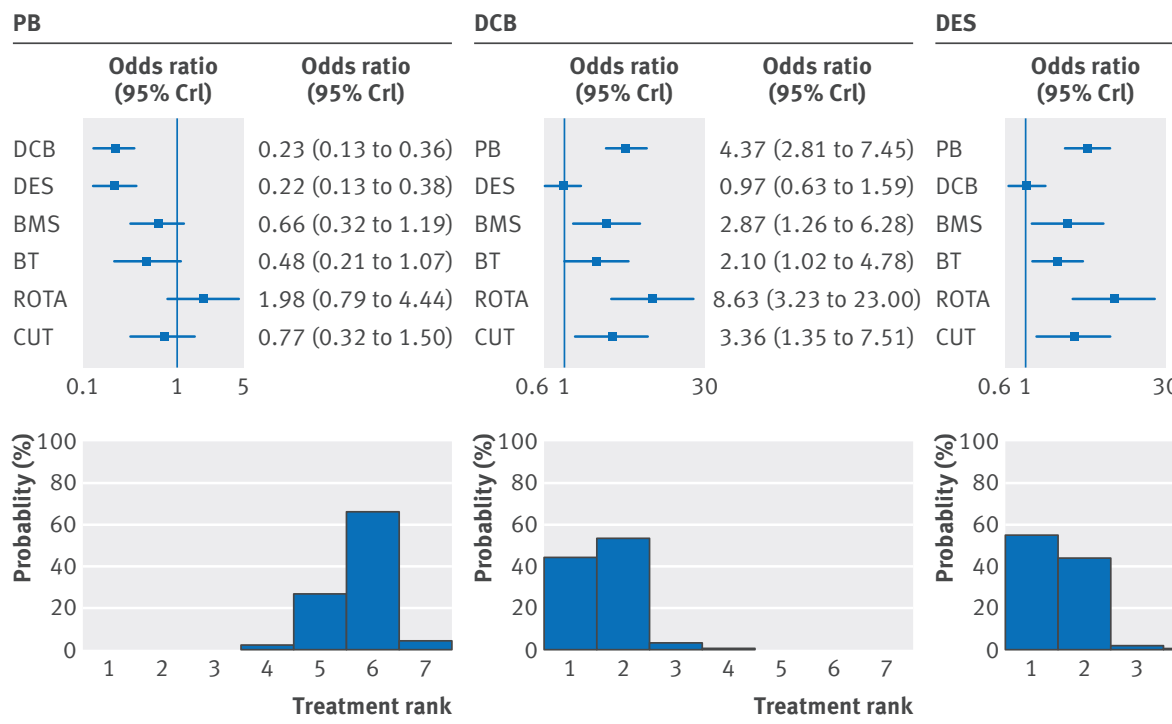

BMS
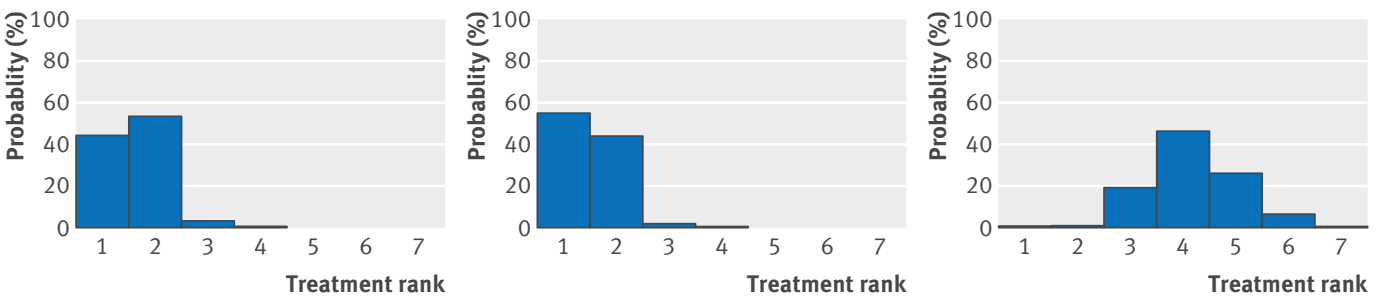

BT
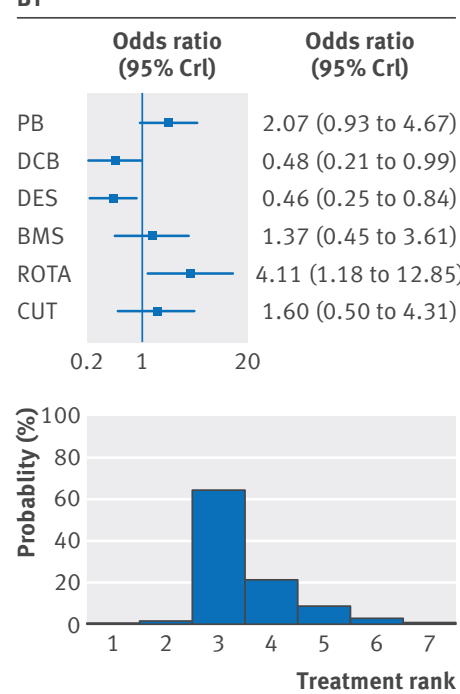

ROTA

Odds ratio
(95\% Crl)

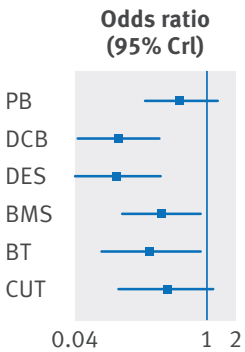

CUT

Odds ratio
(95\% Crl)

Odds ratio

(95\% Crl)

\begin{tabular}{cc}
\hline $\begin{array}{c}\text { Odds ratio } \\
(95 \% \mathrm{Crl})\end{array}$ & $\begin{array}{c}\text { Odds ratio } \\
(95 \% \mathrm{Crl})\end{array}$
\end{tabular}

4.49 (2.66 to 7.83$) \quad \mathrm{PB}$ 1.03 (0.62 to 1.60$)$ DCB 2.96 (1.19 to 6.55) DES 2.17 (1.19 to 4.04) BT 8.89 (3.10 to 23.97) ROTA 3.44 (1.31 to 7.80 ) CUT

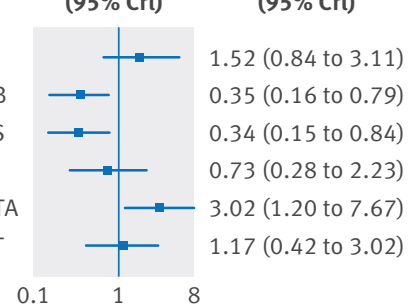

Fig 5 | Effect of interventional treatments on major adverse cardiac events. Forest plots show relative effect of each treatment on major adverse cardiac events as compared with a common reference treatment. Histograms are shown for each treatment reflecting corresponding probabilities for each position in the ranking of the seven strategies (rankograms). $I^{2}$ value $=8.5 \% . P B=$ plain balloon; $D C B=d r u g$ coated balloon; $D E S=d r u g$ eluting stent; $\mathrm{BMS}=$ bare metal stent; $\mathrm{BT}=$ brachytherapy; $\mathrm{ROTA}=$ rotational atherectomy; $\mathrm{CUT}=$ cutting balloon; $\mathrm{OR}=0 \mathrm{dds}$ ratio; $\mathrm{Crl}=\mathrm{credible}$ interval

balloons may be larger in BMS-ISR than in DES-ISR, whereas the effect of drug eluting stents compared with plain balloons was less influenced by restenotic stent type. After comparing drug coated balloons with drug eluting stents in BMS-ISR and DES-ISR, we saw no differences in their respective network meta-analyses. However, the corresponding frequentist, pairwise meta-analysis showed a significant reduction in the risk of target lesion revascularisation associated with the reimplantation of drug eluting stents for DES-ISR.

Figure 11 shows the stratification of trials comparing drug coated balloons with drug eluting stents in standard pairwise meta-analyses according to the generation of drug eluting stent implanted for in-stent restenosis. Risk of target lesion revascularisation was similar between first generation drug eluting stents and drug coated balloons. However, the risk decreased consistently in the analysis of second generation drug eluting stents versus drug coated balloons, where repeated stenting with everolimus eluting stents was associated with a trend towards a $65 \%$ risk reduction $(\mathrm{P}=0.052)$.

In the study removal analysis, the PEPCAD II trial appeared to unduly influence the pooled estimate of target lesion revascularisation for the comparison of drug eluting stents versus drug coated balloons. After exclusion of PEPCAD II from the main analysis, use of drug eluting stents for in-stent restenosis was associated with a larger reduction in target lesion revascularisation compared with drug coated balloons. This finding was seen in both the network meta-analysis (summary odds ratio $0.49,95 \%$ credible interval 0.22 to 1.08) and corresponding standard pairwise meta-analysis (0.51, 95\% confidence interval 0.31 to 0.84 ; fig 12 ). 
Death

\begin{tabular}{|c|c|c|c|c|c|c|c|}
\hline \multicolumn{2}{|l|}{ PB } & \multicolumn{2}{|l|}{ DCB } & \multicolumn{2}{|l|}{ DES } & \multicolumn{2}{|l|}{ BMS } \\
\hline $\begin{array}{l}\text { Odds ratio } \\
\text { (95\% Crl) }\end{array}$ & $\begin{array}{l}\text { Odds ratio } \\
\text { ( } 95 \% \mathrm{Crl})\end{array}$ & $\begin{array}{l}\text { Odds ratio } \\
\text { ( } 95 \% \text { Crl) }\end{array}$ & $\begin{array}{l}\text { Odds ratio } \\
\text { (95\% Crl) }\end{array}$ & $\begin{array}{l}\text { Odds ratio } \\
\text { ( } 95 \% \mathrm{Crl})\end{array}$ & $\begin{array}{l}\text { Odds ratio } \\
\text { ( } 95 \% \text { Crl) }\end{array}$ & $\begin{array}{l}\text { Odds ratio } \\
\text { (95\% Crl) }\end{array}$ & $\begin{array}{l}\text { Odds ratio } \\
\text { (95\% Crl) }\end{array}$ \\
\hline
\end{tabular}

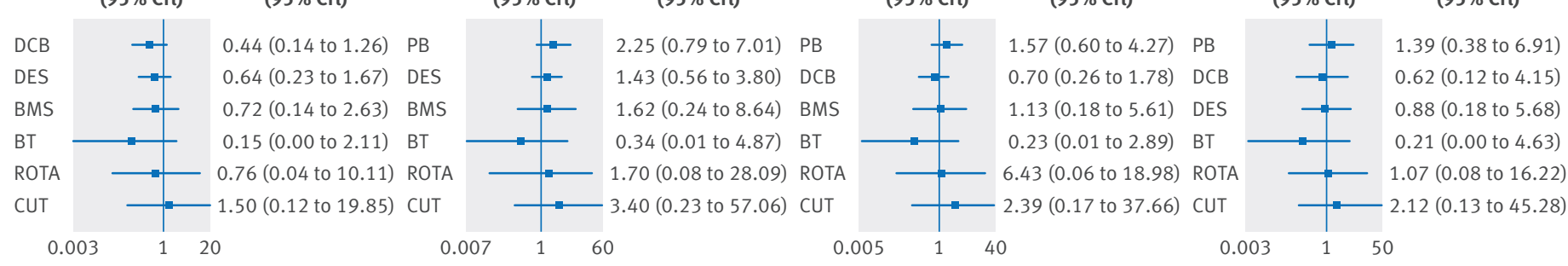

BT ROTA
RT

OTA CUT

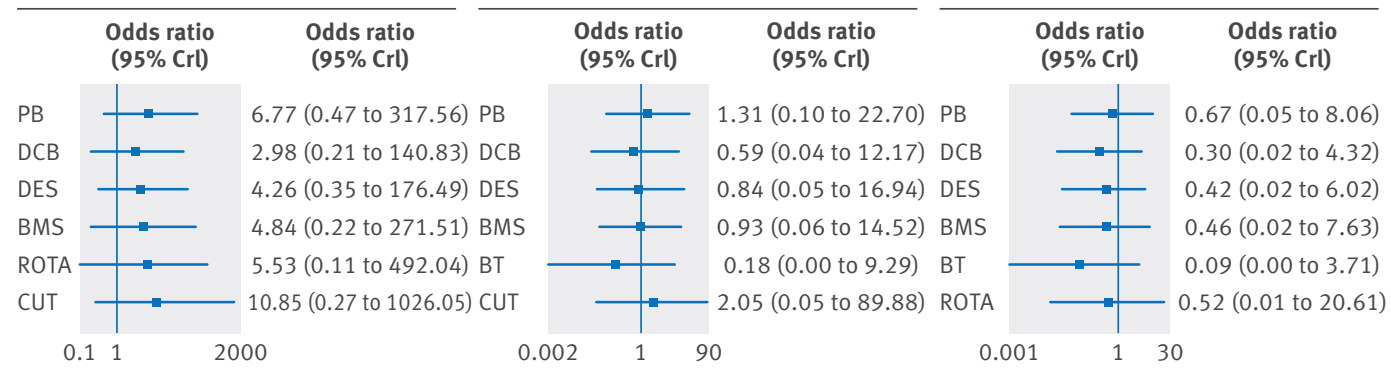

Myocardial infarction

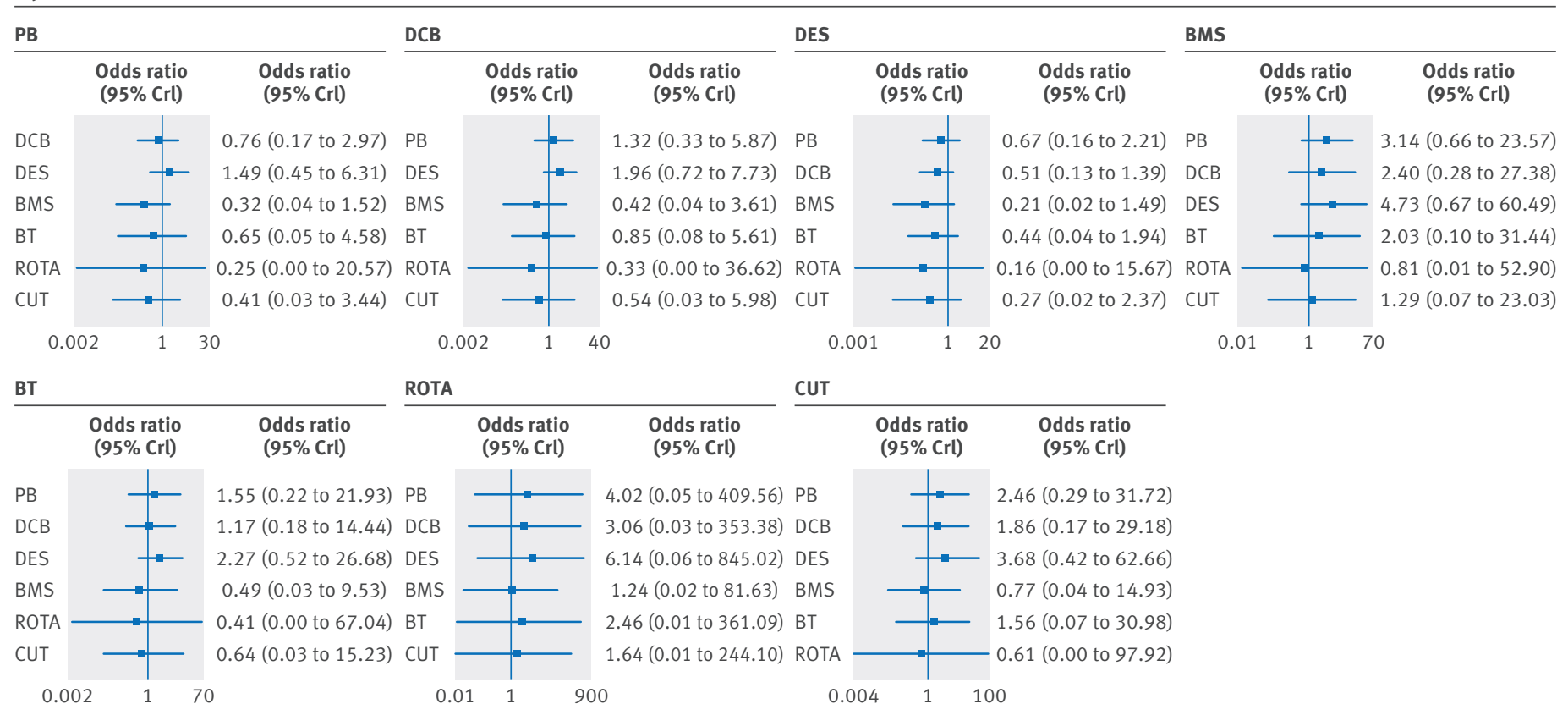

Fig 6 | Effect of interventional treatments on secondary clinical endpoints. Forest plots show relative effect of each treatment on secondary clinical endpoints as compared with a common reference treatment. $\left.\right|^{2}$ values: death=0\%; myocardial infarction=11.8\%. $P B=p l a i n$ balloon; $D C B=d r u g$ coated balloon; $\mathrm{DES}=$ drug eluting stent; $\mathrm{BMS}=$ bare metal stent; $\mathrm{BT}=$ brachytherapy; $\mathrm{ROTA}=$ rotational atherectomy; $\mathrm{CUT}=\mathrm{cutting}$ balloon; $\mathrm{OR}=\mathbf{0}$ dds ratio; $\mathrm{Crl}=$ credible interval

A stent thrombosis network meta-analysis was not feasible due to the low number of events and trials reporting on such endpoint. However, we did standard pairwise meta-analyses for the loop plain balloons, drug coated balloons, and drug eluting stents. No significant differences among treatments were observed (web fig 6).

We also excluded rotational atherectomy and brachytherapy from the main network and applied a study size filter of at least 50 patients per arm in the attempt to minimise possible influences of outdated treatments and smaller trials. The results of this network subanalysis for target lesion revascularisation and late lumen loss did not show substantial variations compared with the conclusions of the main analysis (web fig 7).

\section{Publication bias}

Overall, visual estimation of comparison adjusted funnel plots did not suggest significant asymmetry for all 
Network node-split

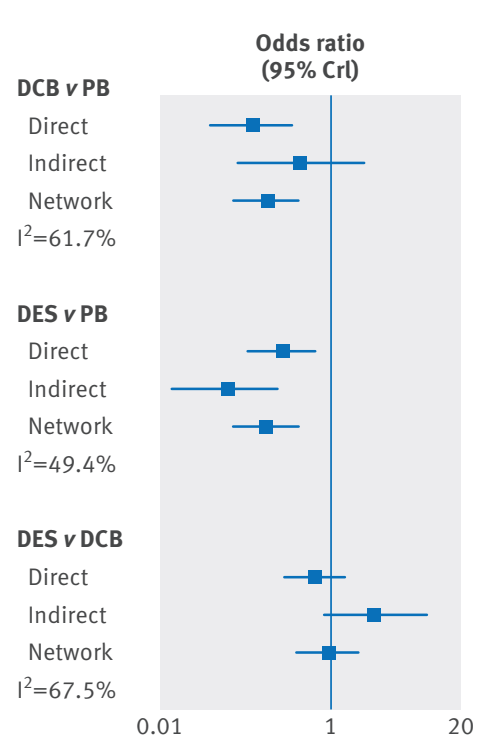

Global I ${ }^{2}=57.2 \%$

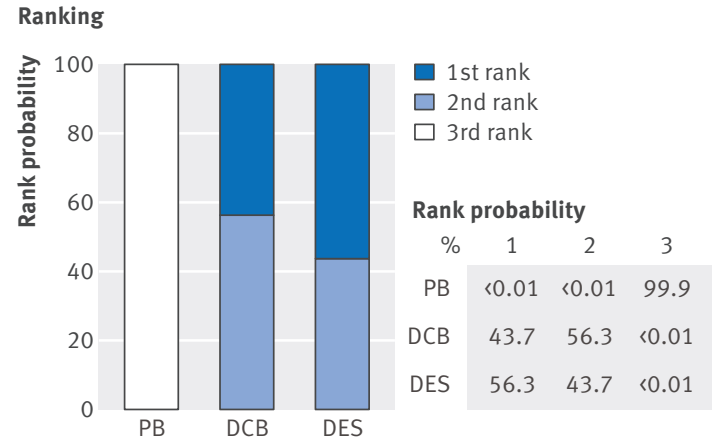

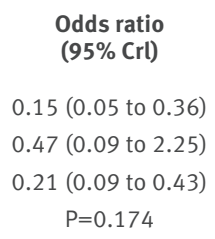

$0.28(0.12$ to 0.67$)$ 0.07 (0.02 to 0.25$)$

0.19 (0.08 to 0.42$)$ $\mathrm{P}=0.066$

$0.64(0.31$ to 1.40$)$ $3.00(0.84$ to 13.15$)$ 0.95 (0.46 to 2.07) $\mathrm{P}=0.043$
Frequentist pair wise subanalyses

Fig 7 | Bayesian network subanalysis of closed loop plain balloons (PB), drug coated balloons (DCB), and drug eluting stents (DES), with additional frequentist pairwise comparisons for the endpoint of target lesion revascularisation. Network of trials investigating the effects of each treatment was considered. Left section of figure shows network node-split (network subanalysis) and corresponding rank probabilities for target lesion revascularisation; direct evidence estimates represent results of the Bayesian pairwise meta-analyses, and $\mathrm{I}^{2}$ values for each comparison grade the heterogeneity between trials from a Bayesian estimate of $\tau^{2}$. Right section of figure shows standard, frequentist pairwise comparisons from the DerSimonian-Laird random effect model. $\mathrm{OR}=$ odds ratio; $\mathrm{Crl}=$ credible interval; $\mathrm{Cl}=$ confidence interval

\section{No of events/total}

Study

DCB PB

Habara et al 2011

PACCOCATH ISR I/II

$1 / 25$

$11 / 72 \quad 14 / 38$

PEPCAD DES

ISAR DESIRE 3

Habara et al 2013

$30 / 137 \quad 56 / 134$

$4 / 136 \quad 22 / 71$

$48 / 424 \quad 122 / 322$

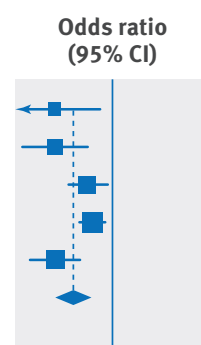

$\begin{array}{cc}\text { Weight } & \text { Odds ratio } \\ (\%) & (95 \% \mathrm{Cl})\end{array}$

10.80 .06 (0.01 to 0.54$)$

16.20 .07 (0.01 to 0.30 )

23.50 .31 (0.12 to 0.78)

$28.50 .39(0.23$ to 0.66$)$

$21.0 \quad 0.07$ (0.02 to 0.21$)$

$100.0 \quad 0.16(0.07$ to 0.38$)$

$P<0.001$

$\begin{array}{lllll}0.01 & 0.1 & 1 & 10 & 100\end{array}$

\begin{tabular}{|c|c|c|c|c|c|}
\hline Study & DES & PB & $\begin{array}{c}\text { Odds ratio } \\
(95 \% \mathrm{Cl})\end{array}$ & $\begin{array}{c}\text { Weight } \\
\text { (\%) }\end{array}$ & $\begin{array}{c}\text { Odds ratio } \\
(95 \% \mathrm{Cl})\end{array}$ \\
\hline ISAR DESIRE & $27 / 200$ & $33 / 100$ & & 37.8 & 0.32 (0.18 to 0.57$)$ \\
\hline RIBS II & $8 / 76$ & $22 / 74$ & & 16.3 & 0.28 (0.11 to 0.67$)$ \\
\hline CRISTAL & $8 / 136$ & $8 / 61$ & & 12.0 & $0.41(0.15$ to 1.16$)$ \\
\hline ISAR DESIRE 3 & $17 / 131$ & $56 / 134$ & & 33.9 & 0.21 (0.11 to 0.38$)$ \\
\hline Total $(95 \% \mathrm{Cl})$ & $60 / 543$ & $119 / 369$ & & 100.0 & 0.28 (0.19 to 0.40$)$ \\
\hline \multicolumn{3}{|c|}{ Random effects model: $\tau^{2}=0, P=0.642, I^{2}=0 \%$} & & & $P<0.001$ \\
\hline
\end{tabular}

Study

No of events/total

PEPCAD ॥

ISAR DESIRE 3

PEPCAD ISR China

RIBS V

SEDUCE

RIBS IV

Total $(95 \% \mathrm{Cl})$

DES DCB

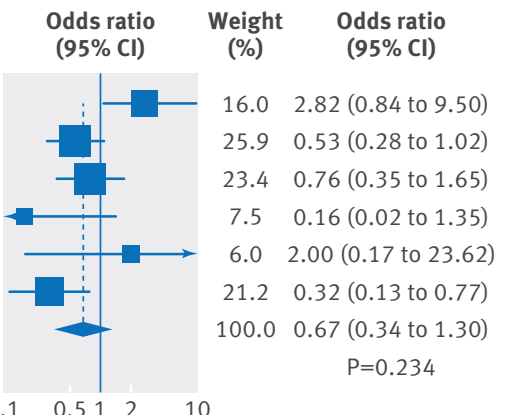

$0.1 \quad 0.512 \quad 10$ endpoints. However, a consistent number of trials (almost all involving drug coated balloons) fell outside the significance boundaries in the late lumen loss analysis (web figs 8 and 9). Contour enhanced funnel plots for the comparison of drug eluting stents versus drug coated balloon were implemented by Peters' test (target lesion revascularisation) and Egger's test (late lumen loss). These plots did not outline significant publication bias (web figs 10 and 11). However, for late lumen loss, this non-significance could be due to the limited number of trials, because visual inspection suggested an asymmetric distribution and the Egger's test $P$ value of 0.127 was close to the formal significance threshold of 0.10 .

\section{Discussion}

This updated network meta-analysis comparing all available treatments for in-stent restenosis had four main findings. Firstly, drug coated balloons and drug eluting stents are the most effective interventional treatments for in-stent restenosis compared with other currently available strategies, leading to superior and long term efficacy in terms of clinical, angiographic and antirestenotic outcomes. Secondly, use of a plain balloon alone is significantly less effective than a drug coated balloon or a drug eluting stent. Thirdly, drug coated balloons might exert a larger efficacy in BMS-ISR than in DES-ISR. Finally, second generation everolimus eluting stents have shown a tendency to reduce the risk of target lesion revascularisation compared with drug coated balloons.

Recently, a meta-analysis by Lee and colleagues evaluated plain balloons, drug coated balloons, and drug eluting stents in the treatment of in-stent restenosis, suggesting drug coated balloons to be the best therapy. ${ }^{41}$ Our meta-analysis differs from that earlier study in several ways.

Firstly, the main objective of our study was the evaluation of all existing interventional strategies for in-stent restenosis using data from randomised trials, whereas Lee and colleagues' study included only three 
Network node-split

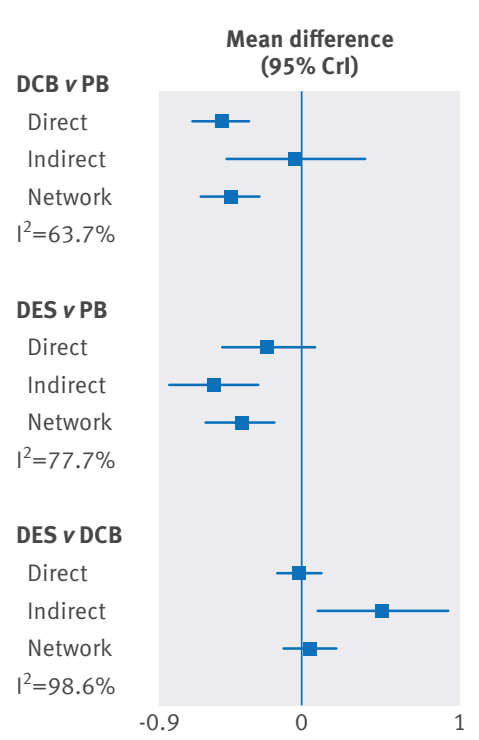

Global I ${ }^{2}=96.6 \%$

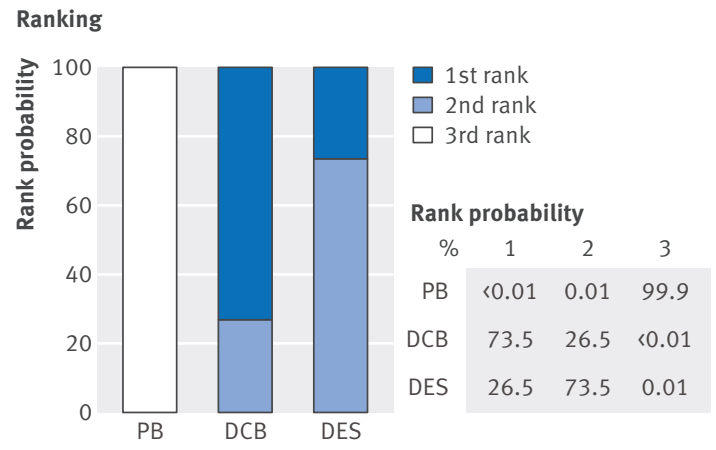

Frequentist pair wise subanalyses

$\begin{gathered}\text { Mean difference } \\ (95 \% \text { Crl })\end{gathered}$
$-0.50(-0.69$ to -0.33$)$
$-0.03(-0.47$ to 0.40$)$
$-0.44(-0.63$ to -0.27$)$
$P=0.049$
$-0.22(-0.50$ to 0.07$)$
$-0.56(-0.83$ to -0.29$)$
$-0.39(-0.60$ to -0.18$)$
$P=0.073$

$-0.02(-0.15$ to 0.13$)$
$0.51(0.10$ to 0.92$)$
$0.05(-0.11$ to 0.22$)$
$P=0.036$

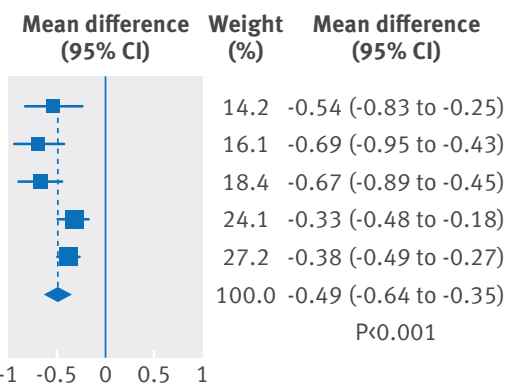

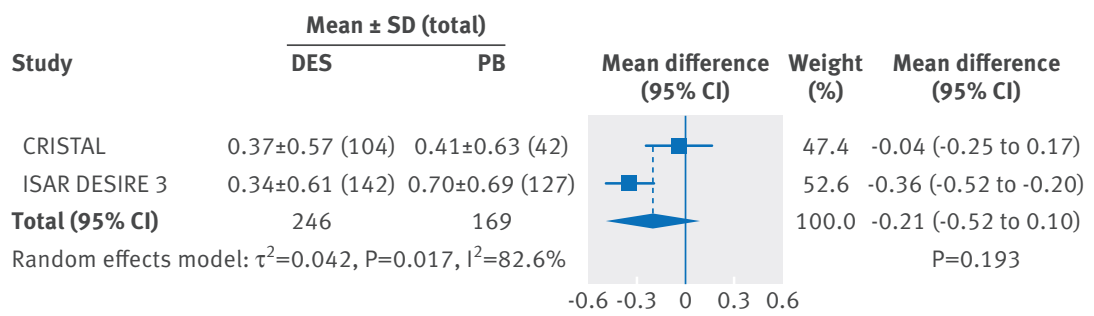

Fig 8 | Bayesian network subanalysis of closed loop plain balloons (PB), drug coated balloons (DCB), and drug eluting stents (DES), with additional frequentist pairwise comparisons for the endpoint of late lumen loss. Network of trials investigating the effects of each treatment was considered. Left section of figure shows network node-split (network subanalysis) and corresponding rank probabilities for target lesion revascularisation; direct evidence estimates represent results of the Bayesian pairwise meta-analyses, and $I^{2}$ values for each comparison grade the heterogeneity between trials from a Bayesian estimate of $\tau^{2}$. Right section of figure shows standard, frequentist pairwise comparisons from the DerSimonian-Laird random effect model. $\mathrm{MD}=$ =mean difference; $\mathrm{Crl}=$ credible interval; $\mathrm{Cl}=$ confidence interval; $\mathrm{SD}=$ standard deviation

treatments. Secondly, the previous meta-analysis did not include two of the six available randomised trials comparing drug coated balloons with drug eluting stents (SEDUCE, RIBS IV), which could explain the disparity between our results and their conclusions. Thirdly, we also performed standard pairwise comparisons between treatment arms and multiple subanalyses to critically complement the results of the network meta-analysis. Finally, we also considered angiographic endpoints whereas the study by Lee and colleagues focused essentially on clinical outcomes.

\section{Use of drug coated balloons versus drug eluting} stents for coronary in-stent restenosis

Intracoronary imaging indicates a leading role of exuberant neointimal proliferation among the potential mechanisms of in-stent restenosis. ${ }^{42}$ Use of drug coated balloons is an emerging treatment for in-stent restenosis, with the putative advantage of delivering an antiproliferative treatment without adding a second layer of metal. ${ }^{4}$ Our study showed that compared with plain balloons, drug coated balloons resulted in reductions of $79 \%$ relative risk of target lesion revascularisation and $0.44 \mathrm{~mm}$ mean difference in late lumen loss. However, in-stent restenosis has been also associated with stent underexpansion (that is, insufficient stent expansion at implantation or chronic recoil), uneven stent struts disposition in complex lesions, and neoatherosclerosis. ${ }^{43} 44$ These factors could theoretically disfavour drug coated balloons, a treatment that cannot guarantee a constant radial strength. ${ }^{11} 43$

In-stent implantation of drug eluting stents, providing additional permanent scaffolding inside the restenotic stent, ${ }^{45}$ could overcome the mechanical limitations of drug coated balloons. Although the trials in our systematic review showed no significant differences in periprocedural complications and treatment crossover between drug coated balloons and drug eluting stents, repeated 
Network node-split

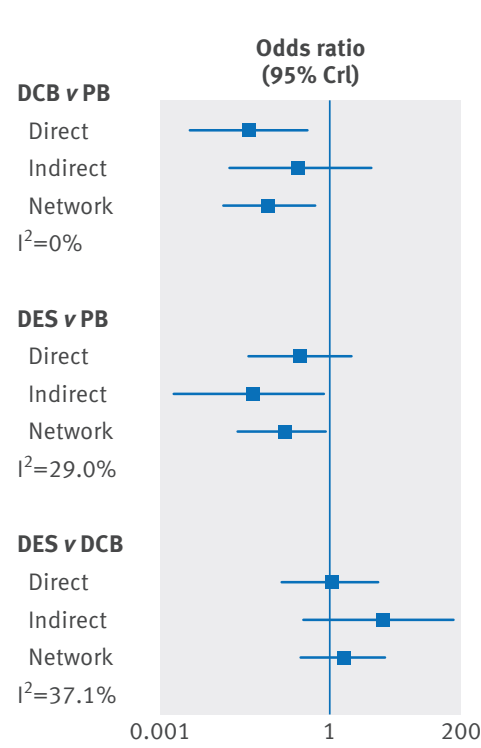

Frequentist pair wise subanalyses

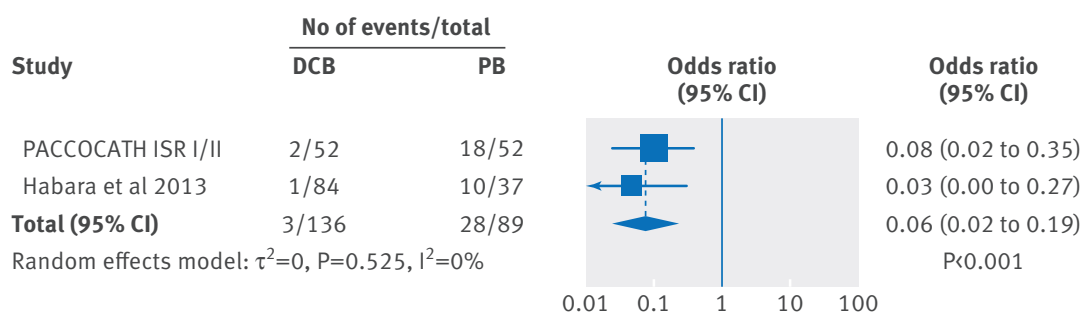

0.30 (0.04 to 2.30$)$

0.05 (0.01 to 0.76$)$

0.19 (0.03 to 0.87 )

$\mathrm{P}=0.216$

1.11 (0.15 to 6.89$)$

6.93 (0.35 to 182.08$)$

1.82 (0.33 to 9.22$)$

$\mathrm{P}=0.212$

\begin{tabular}{lcc} 
& \multicolumn{2}{c}{ No of events/total } \\
\cline { 2 - 3 } Study & DES & PB \\
& & \\
ISAR DESIRE & $27 / 200$ & $33 / 100$ \\
RIBS II & $8 / 76$ & $22 / 74$ \\
Total $(95 \% \mathrm{CI})$ & $35 / 276$ & $55 / 174$ \\
Random effects model: $\tau^{2}=0, \mathrm{P}=0.809, \mathrm{I}^{2}=0 \%$
\end{tabular}

Odds ratio

(95\% CI)

0.32 (0.18 to 0.57$)$

0.28 (0.11 to 0.67$)$

$0.30(0.19$ to 0.50$)$

Global I ${ }^{2}=45.3 \%$

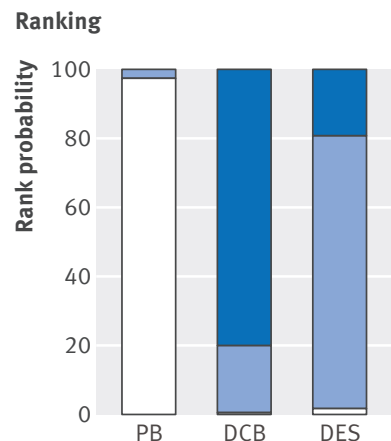

\begin{tabular}{|c|c|c|c|}
\hline \multicolumn{4}{|c|}{$\begin{array}{l}\square \text { 1st rank } \\
\square \text { 2nd rank } \\
\square \text { 3rd rank }\end{array}$} \\
\hline \multicolumn{4}{|c|}{ Rank probability } \\
\hline$\%$ & 1 & 2 & 3 \\
\hline 10 & 0.3 & 2.1 & 97.6 \\
\hline $\mathrm{DCB}$ & 80.3 & 19.1 & 0.6 \\
\hline DES & 19.4 & 78.8 & 1.8 \\
\hline
\end{tabular}

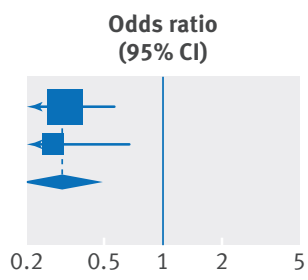

$P<0.001$

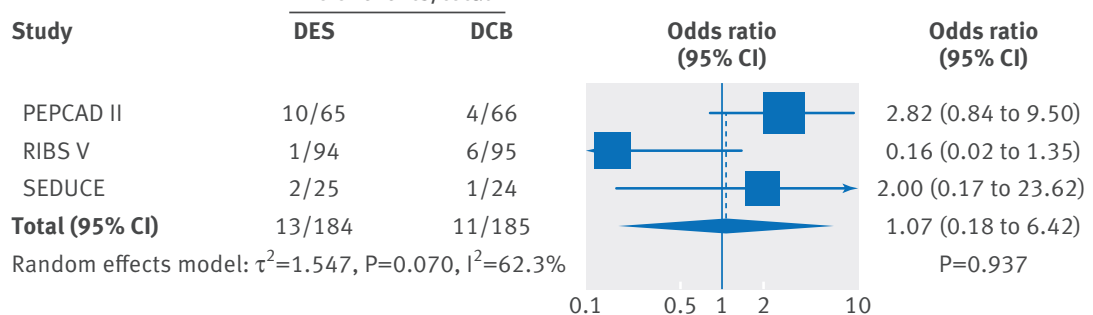

Fig 9 Subgroup analysis of antirestenotic efficacy of plain balloons, drug coated balloons, and drug eluting stents, according to BMS-ISR. Network

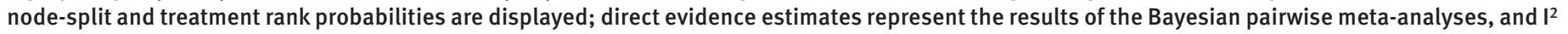

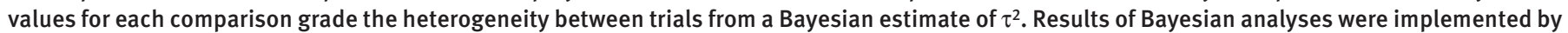
frequentist, random effect, pairwise comparisons. $\mathrm{OR}=$ odds ratio; $\mathrm{Crl}=$ credible interval; $\mathrm{Cl}=$ confidence interval

stenting with drug eluting stents might be a more reasonable indication for relevant periprocedural tissue protrusion and in-stent or stent edge dissection. However, small vessel size and diabetes trigger further neointimal proliferation, and an additional metallic layer could critically amplify the risk of recurrent in-stent restenosis. ${ }^{46} 47$ Selection of the best revascularisation strategy between drug coated balloons and drug eluting stents should be therefore tailored on a case by case basis according to lesion and patient characteristics.

The similar antirestenotic efficacy of drug coated balloons and drug eluting stents observed in our meta-analysis could result from the trade-off between advantages and shortcoming of the two devices. Drug coated balloons are associated with worse acute angiographic results, but are more respectful of the original coronary anatomy, induce lower vascular inflammatory response, and exert a lower stimulus to endothelial growth in the long term. Drug eluting stents guarantee a larger, immediate minimum lumen diameter and more predictable acute effects at the risk of reiterating the process of neointimal growth. ${ }^{45} 48$ The most relevant concern about drug coated balloons is the durability of the antirestenotic effect, because local drug use in the short term may not result in a longlasting inhibition of restenosis. However, the putative angiographic and clinical superiority of drug eluting stents in the long term for in-stent restenosis is presently not supported by a solid evidence basis.

Our meta-analysis suggests that second generation everolimus eluting stents might be the best strategy for in-stent restenosis. In agreement with large and unselected observational studies, ${ }^{49}{ }^{50}$ our stratified analysis showed that these second generation stents produced an almost significant $65 \%$ reduction in the risk of target lesion revascularisation compared with drug coated balloons. The efficacy of drug coated balloons and first generation stents was comparable. However, it is still unknown whether this possible benefit is generalisable to diffuse and neoatherosclerotic in-stent restenosis, especially if recurrent.

The available data do not allow for comparison between drug coated balloons and drug eluting stents according to first or recurrent in-stent restenosis, and 
Network node-split

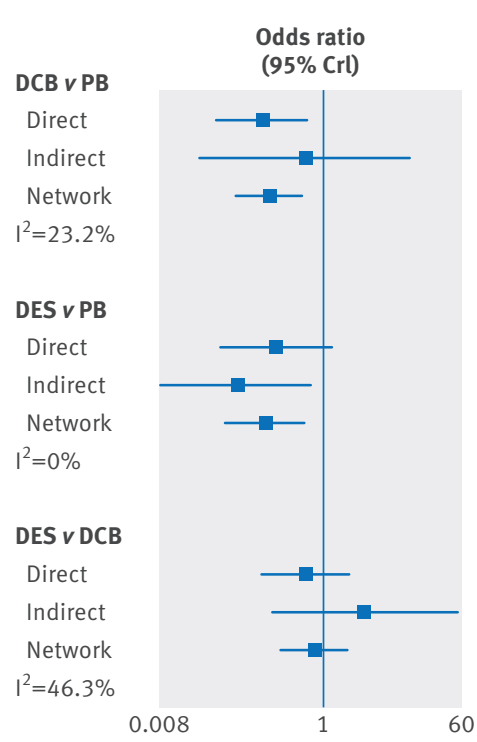

Global I ${ }^{2}=44.3 \%$

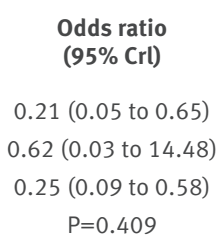

0.27 (0.05 to 1.48$)$

0.10 (0.01 to 0.77$)$

0.20 (0.06 to 0.55$)$

$\mathrm{P}=0.338$

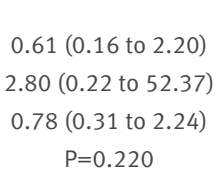

\section{Ranking}

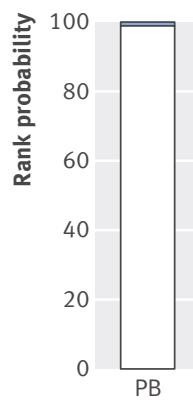

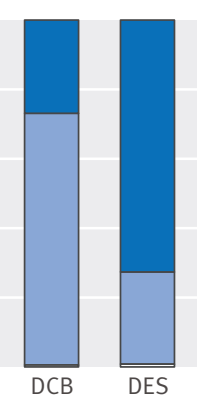

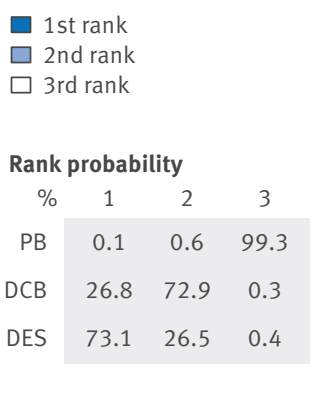

Frequentist pair wise subanalyses

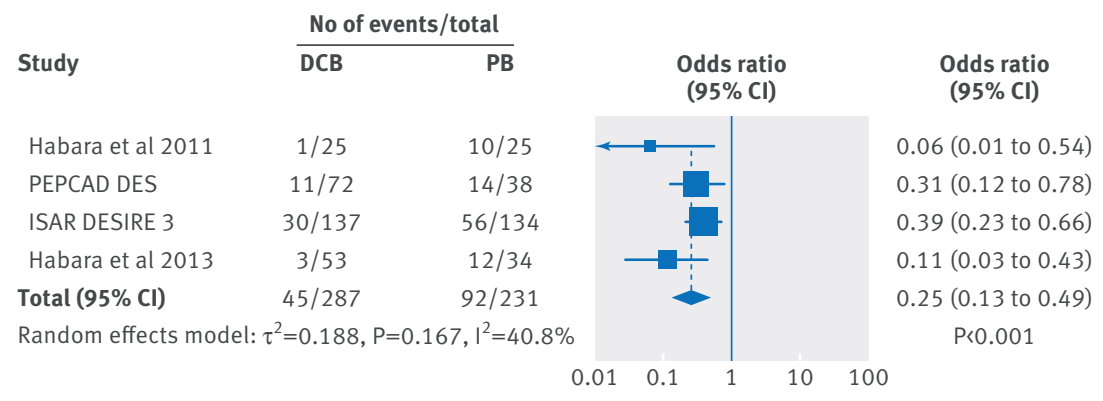

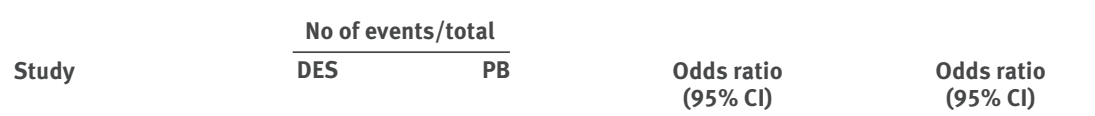

0.41 (0.15 to 1.16$)$

0.21 (0.11 to 0.38$)$

0.26 (0.14 to 0.48$)$

$P<0.001$

Fig 10 | Subgroup analysis of antirestenotic efficacy of plain balloons, drug coated balloons, and drug eluting stents, according to DES-ISR. Network node-split and treatment rank probabilities are displayed; direct evidence estimates represent the results of the Bayesian pairwise meta-analyses, and $\mathrm{I}^{2}$ values for each comparison grade the heterogeneity between trials from a Bayesian estimate of $\tau^{2}$. Results of Bayesian analyses were implemented by frequentist, random effect, pairwise comparisons. $\mathrm{OR}=$ odds ratio; $\mathrm{Crl}=$ credible interval; $\mathrm{Cl}=$ confidence interval

selection of the best strategy for patients with resistant in-stent restenosis (that is, at least three instances) remains uncertain. Owing to a lack of evidence, we could not establish whether implantation of drug eluting stents for recurrent in-stent restenosis is better than repeat use of drug coated balloons after a failed drug coated balloon strategy. However, the first option seems to be a rational second line treatment. ${ }^{50}$ Overall, an initial approach to in-stent restenosis with drug coated balloons might be reasonable, especially in BMS-ISR and in patients with small vessels or diabetes. Second generation drug eluting stents should then be prioritised for DES-ISR and complex in-stent restenosis. Because patients with resistant in-stent restenosis tend to have multiple recurrent events, coronary artery bypass grafting should be also considered after multiple failures of percutaneous coronary intervention. ${ }^{21}$

In view of the anatomical variables potentially influencing early and late procedural antirestenotic efficacy depending on the selection of drug coated balloons or drug eluting stents, both treatments should be considered quick, safe, and effective for non-complex instances of coronary in-stent restenosis. However, in angiographic and unstable lesions and clinical patterns, implantation of drug eluting stents could be preferable owing to the superior mechanical guarantees of metallic scaffolding. In addition, the decision between these two treatments could be affected by varying postprocedural pharmacological management. ${ }^{48}$ Second generation drug eluting stents have shown to be more biocompatible and less thrombogenic, hence allowing for only six months of dual antiplatelet therapy in de novo lesions. ${ }^{51}$ However, drug coated balloons deliver locally the antiproliferative medication and avoid permanent structures inside the vessel that need prolonged antithrombotic coverage. Although planned duration of dual antiplatelet therapy varies widely in randomised trials with drug coated balloons (including de novo lesions, in-stent restenosis, or peripheral artery disease), manufacturers advise three months of therapy following use of drug coated balloons. In some trials investigating the efficacy of drug coated balloons for in-stent restenosis or small vessel lesions, one month of dual antiplatelet therapy did not show any safety 
First generation drug eluting stent

\begin{tabular}{|c|c|c|c|}
\hline \multirow[b]{2}{*}{ Study } & \multicolumn{2}{|c|}{ No of events/total } & \multirow[b]{2}{*}{$\begin{array}{l}\text { Odds ratio } \\
\text { ( } 95 \% \mathrm{Crl})\end{array}$} \\
\hline & DES & DCB & \\
\hline PEPCAD II & $10 / 65$ & $4 / 66$ & \\
\hline ISAR DESIRE 3 & $17 / 131$ & $30 / 137$ & \\
\hline PEPCAD ISR China & $13 / 106$ & $17 / 109$ & \\
\hline Total $(95 \% \mathrm{Cl})$ & $40 / 302$ & $51 / 312$ & \\
\hline Random effects mo & del: $I^{2}=6$ & $.6 \%$ & \\
\hline
\end{tabular}

$$
\begin{array}{lllll}
0.2 & 0.5 & 1 & 2 & 5
\end{array}
$$

Second generation drug eluting stent

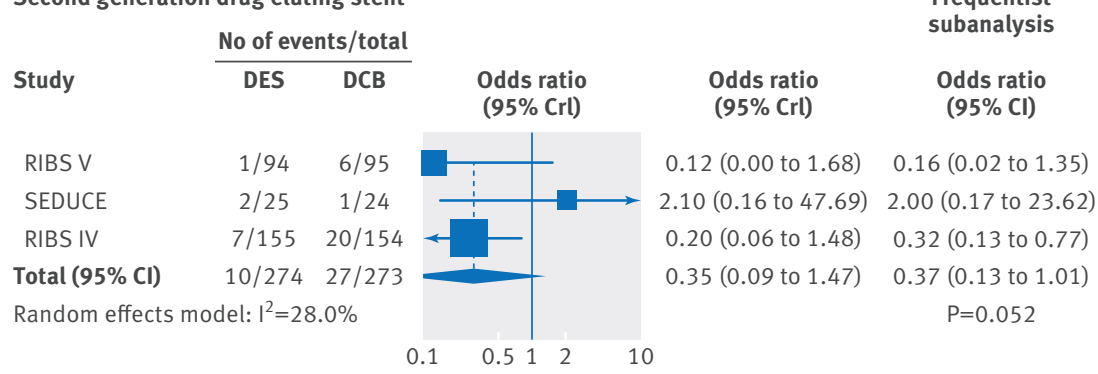

Fig 11 | Risk of target lesion revascularisation between use of first generation or second generation drug eluting stents and drug coated balloons for in-stent restenosis. Bayesian results were implemented by frequentist analyses. $D C B=d r u g$ coated balloon; $D E S=d r u g$ eluting stent; $\mathrm{OR}=$ odds ratio; $\mathrm{Cr}=$ credible interval; $\mathrm{Cl}=$ confidence interval

issue. ${ }^{48}$ Therefore, drug coated balloons might be indicated in patients with urgent surgical indications or at high haemorrhagic risk.

\section{Potential sources of inconsistency and heterogeneity}

The need for a meta-analysis comparing drug eluting stents versus drug coated balloons is supported by the absence of robust antirestenotic differences between treatments across trials of in-stent restenosis. An objective of the meta-analysis is to combine non-conclusive evidence from individual trials to strengthen evidence from the comparison of two or more treatments. Importantly, characteristics of original trials (including

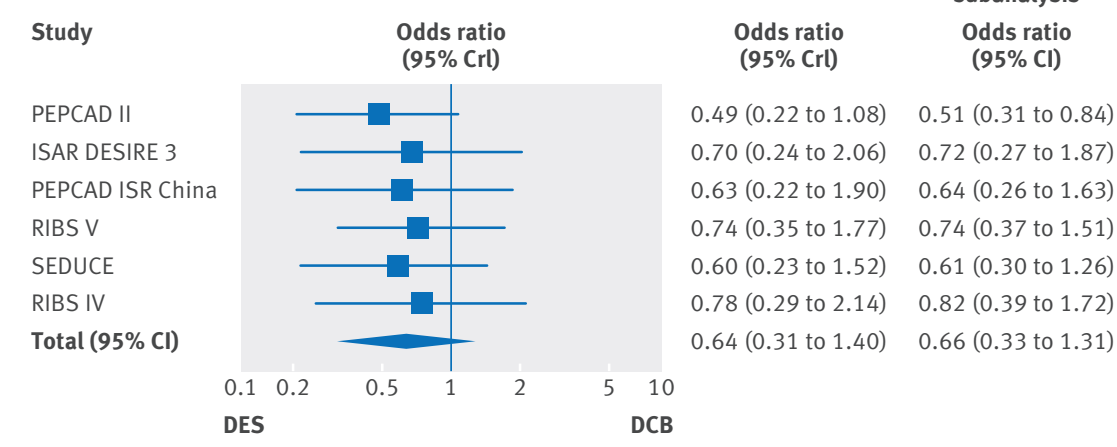

Fig 12 | Risk of target lesion revascularisation after sequential removal of each trial comparing use of drug eluting stents with drug coated balloons for treatment for in-stent restenosis. One trial was removed at a time from the overall analysis to define its individual impact on the pooled risk of target lesion revascularisation. Bayesian results were implemented by frequentist analyses. $\mathrm{DCB}=$ drug coated balloon; $\mathrm{DES}=\mathrm{drug}$ eluting stent; $\mathrm{OR}=$ odds ratio; $\mathrm{Cr}=$ credible interval; $\mathrm{Cl}=$ confidence interval design, eligibility criteria, clinical and procedural features) can make the results of indirect comparisons quite different from those of direct comparison. The significant incoherence between direct and indirect effects is known as inconsistency.

We attempted to reduce potential sources of inconsistency affecting the indirect evidence (that is, obsolete treatments, cutting balloon node) by restricting our analysis to the smallest network of plain balloons, drug coated balloons, and drug eluting stents. A network meta-analysis can identify external influences on the mean effect of a specific comparison that otherwise would have remained unreported with standard pairwise meta-analytical methods. However, even in the smallest network subanalysis, the inconsistency remained unchanged and significant Bayesian $P$ values were observed.

Therefore, the conflict between direct and indirect evidence in the comparison of drug coated balloons versus drug eluting stents could have two explanations that are not mutually exclusive. Firstly, if we assume the direct evidence (which numerically favoured drug eluting stents) to be true, one possible explanation to the indirect evidence trending in the opposite direction is that trials comparing drug coated balloons with plain balloons overstate the efficacy of drug coated balloons, or trials comparing drug eluting stents with plain balloons understate the efficacy of drug eluting stents. In our analysis, we detected a high heterogeneity in the comparison of drug coated balloons versus plain balloons, with two smaller trials showing larger treatment effects.

Secondly, if we assume the indirect evidence (which numerically disfavoured drug eluting stent) to be true, a possible explanation is a significant intrinsic inconsistency in the comparison of drug coated balloons with drug eluting stents. In the individual removal analysis, results were in line with this hypothesis, showing that the risk of target lesion revascularisation tended to be influenced by the oldest trial (PEPCAD II). Conversely, the inconsistency for late lumen loss could have been driven by very different, trial specific, mean estimates ranging from 0.04 to $0.55 \mathrm{~mm}$ for drug eluting stents and from 0.14 to $0.46 \mathrm{~mm}$ for drug coated balloons (same device).

\section{Other treatments for coronary in-stent restenosis}

Our results support the notion that bare metal stents and brachytherapy should be no longer considered in the contemporary management of in-stent restenosis. Indeed, although in-stent implantation of bare metal stents for in-stent restenosis provides higher acute lumen gain and better procedural results than plain balloons, it leads to a comparable minimum lumen diameter and percent diameter stenosis after only six months, largely due to significantly higher levels of late lumen loss..$^{52}$ In addition, at six to 12 months, bare metal stents were associated with the highest mean difference in late lumen loss. Several trials in this network meta-analysis concluded that vascular brachytherapy is less effective in treating in-stent restenosis than drug eluting stents. We included brachytherapy in our 
network meta-analysis to provide an additional reference for more effective strategies in the network, and to quantify a pooled estimate of the antirestenotic effect of this treatment compared with drug coated balloons and drug eluting stents.

This meta-analysis also underlines that rotational atherectomy cannot be considered a standalone treatment for in-stent restenosis, because its outcomes are significantly inferior than those observed with the other treatments. Still, this strategy could be considered as complementary and preliminary to the implantation of drug eluting stents in old, hard, and calcified lesions caused by in-stent restenosis. ${ }^{54} 55$ Importantly, although rotational atherectomy for in-stent restenosis could be associated with a higher incidence of mechanical complications (that is, stent fracture, coronary perforation, and plaque embolisation), we did not observe a significant increase in death and myocardial infarction at follow-up.

Cutting balloon use was associated with a higher risk of target lesion revascularisation and higher mean difference in late lumen loss than use of a drug coated balloon or drug eluting stent. We did not evaluate the effects of the newer scoring balloon in this meta-analysis because data from randomised trials were not available. However, results from the ISAR-DESIRE 4 trial (NCT01632371) and other reports exploring the use of scoring balloons to prepare in-stent restenosis lesions for drug coated balloons are pending.

\section{Future perspectives}

Future direction of in-stent restenosis treatment will depend from the results of additional and larger randomised trials currently comparing drug coated balloons with second generation drug eluting stents. The ongoing DARE (NCT01127958) and TIS (NCT01735825) trials are investigating the superiority of second generation everolimus eluting stents over drug coated balloons. In the MAGIC-TOUCH trial (NCT02400632), researchers are investigating a new sirolimus coated balloon; these results will provide an additional reference for current types of drug coated balloons that elute paclitaxel.

Isolated initial reports have suggested that bioresorbable scaffolds may offer sufficient mechanical support as well as reduced neointimal proliferative stimuli in in-stent restenosis. ${ }^{56}{ }^{57}$ However, unfavourable properties of current bioresorbable scaffolds (that is, thick struts, lower radial force than drug eluting stents, and narrower margins for overexpansion after deployment ${ }^{58}$ ) could frustrate this approach, which warrants validation in targeted investigations in a sufficient number of patients.

\section{Limitations}

The results of our meta-analysis should be interpreted taking the following limitations into account. Firstly, a meta-analysis shares the limitations of the studies included. To minimise this unavoidable shortcoming, only randomised trials were included. The qualitative bias assessment did not suggest significant causes of concern, and differences in follow-up completeness were trivial as the consequence of the strict angiographic surveillance scheduled in all studies. However, we noticed that studies had different proportions of diabetes mellitus, focal or diffuse angiographic patterns in in-stent restenosis, and different minimum lumen diameters at baseline, which cannot be fully appreciated at the study level.

Furthermore, the trials covered a period of about 15 years, which could have introduced unmeasured differences among treatments. Almost all studies were of open label design; this was an unavoidable consequence of the different constitutive characteristics of the devices under investigation, which do not consent complete masking to the operator, with few exceptions (plain balloons and drug coated balloons in PACCOCATH ISR I/II).

The absence of long term follow-up and the varying periods of investigation across trials were also limitations. There are insufficient data on clinical and angiographic outcomes several years after in-stent restenosis treatment, and almost all the studies included in our meta-analysis did not provide information beyond 12 months.

We were forced to select summary odds ratios as an outcome measure owing to the trials not providing enough information to indirectly calculate hazard ratios. However, we compared estimated summary odds ratios with hazard ratios from individual patient data from trials reporting those values, and found that the difference between the two outcome measures was trivial. This lack of difference could have been a result of the favourable follow-up length $(\leq 12$ months) and the fact that very few patients were lost at follow-up.

Although this meta-analysis included nearly 5000 patients, some trials were small, of which a large number were powered only for a specific angiographic endpoint, mainly late lumen loss. However, sensitivity analyses on target lesion revascularisation and late lumen loss that were restricted to newer and larger studies did not show relevant deviations from the main results. Additionally, although we observed a numerical distribution for myocardial infarction favouring some treatments over the others, the event rate was low and did not allow us to draw robust conclusions for this endpoint. For similar reasons, the network meta-analysis for stent thrombosis was not feasible because most of the included trials did not report the number of stent thrombosis events, and the remaining reported a low incidence.

Some trials were not included in all analyses because they did not have the specific endpoint of interest or report angiographic values as median and interquartile range. In addition, although few older trials reported only target vessel revascularisation, we pooled these events with target lesion revascularisation, and events of the drug eluting stent group of the ISAR DESIRE trial were derived from collapsing the original groups using sirolimus eluting stents and paclitaxel eluting stents. 
Subgroup analyses according to the type of restenotic stent-either bare metal or drug eluting-could not benefit from randomisation in all instances. The trial by Habara and colleagues (2013) included both devices, and in the PACCOCATH ISR I/II trial (which was included in the subgroup analysis for BMS-ISR), 4\% of patients actually had DES-ISR. We did not stratify groups by first or recurrent in-stent restenosis because the original data did not allow a clear identification of such variants.

Unrelated mean effect models are not well suited to handle trials with more than two arms. Node-split, the most powerful tool to detect inconsistency, is limited to closed loops. However, our meta-analysis included only one three arm trial (ISAR DESIRE 3), and the most effective treatments were all part of closed loops. Finally, our meta-analysis investigated the efficacy of first generation and second generation everolimus eluting stents, but questions regarding the efficacy of newer stents (that is, second and third generations) remain.

\section{Conclusions}

In this network meta-analysis, drug coated balloons and drug eluting stents were shown to be the most effective treatments for in-stent restenosis. Plain balloons, bare metal stents, brachytherapy, rotational atherectomy, and cutting balloons were associated with a higher risk of target lesion revascularisation and inferior angiographic results. We saw no differences in death, myocardial infarction, and stent thrombosis among all included treatments, but such comparisons remain limited by the low number of events. The risk of major adverse cardiac events was consistently reduced with drug coated balloons and drug eluting stents, driven by target lesion revascularisation. Although the main analysis suggested a similar efficacy of drug coated balloons and drug eluting stents, an exploratory subgroup analysis indicated a trend towards a risk reduction in target lesion revascularisation with second generation everolimus eluting stents.

Contributors: DG conceived and designed the study; DG, GG, and PA collected and abstracted the data; DG undertook the statistical analysis; DG, GG, and DC drafted the manuscript; all authors had full access to all the data, including statistical reports and tables; all authors analysed and interpreted the data; all authors critically revised the manuscript for important intellectual content; DC is the guarantor.

Funding: This study received no external funding.

Competing interests: All authors have completed the ICMJE uniform disclosure form at www.icmje.org/coi_disclosure.pdf and declare: no support from any organisation for the submitted work; no financial relationships with any organisations that might have an interest in the submitted work in the previous three years; no other relationships or activities that could appear to have influenced the submitted work.

Ethical approval: None was required.

Data sharing: No additional data available.

The manuscript's guarantor affirms that the manuscript is an honest, accurate, and transparent account of the study being reported, that no important aspects of the study have been omitted and that any discrepancies from the study as planned have been explained.

This is an Open Access article distributed in accordance with the Creative Commons Attribution Non Commercial (CC BY-NC 4.0) license, which permits others to distribute, remix, adapt, build upon this work non-commercially, and license their derivative works on different terms, provided the original work is properly cited and the use is non-commercial. See: http://creativecommons.org/licenses/ by-nc/4.0/.

Stefanini GG, Holmes DR, Jr. Drug-eluting coronary-artery stents. N Engl I Med 2013;368:254-65.

2 Cassese S, Byrne RA, Tada T, et al. Incidence and predictors of restenosis after coronary stenting in 10004 patients with surveillance angiography. Heart 2014;100:153-9.

3 Mauri L, Silbaugh TS, Wolf RE, et al. Long-term clinical outcomes after drug-eluting and bare-metal stenting in massachusetts. Circulation 2008;118:1817-27.

4 Alfonso F, Byrne RA, Rivero F, Kastrati A. Current treatment of in-stent restenosis. J Am Coll Cardiol 2014;63:2659-73.

5 Mehran R, Mintz GS, Popma JJ, et al. Mechanisms and results of balloon angioplasty for the treatment of in-stent restenosis. Am J Cardiol 1996;78:618-22.

$6 \mathrm{Kim}$ YH, Lee BK, Park DW, et al. Comparison with conventional therapies of repeated sirolimus-eluting stent implantation for the treatment of drug-eluting coronary stent restenosis. Am J Cardiol 2006:98:1451-4

7 Alfonso F, Cequier A, Angel J, et al. Restenosis intra-stent balloon angioplasty versus elective stenting I. Am HeartJ 2006;151:e681-9.

8 Kastrati A, Mehilli J, von Beckerath N, et al. Sirolimus-eluting stent or paclitaxel-eluting stent vs balloon angioplasty for prevention of recurrences in patients with coronary in-stent restenosis: a randomised controlled trial. JAMA 2005;293:165-71.

9 Tagliareni F, La Manna A, Saia F, Marzocchi A, Tamburino C. Long-term clinical follow-up of drug-eluting stent restenosis treatment: retrospective analysis from two high volume catheterisation laboratories. Eurolntervention 2010:5:703-8.

10 Rathore S, Kinoshita Y, Terashima M, et al. A comparison of clinical presentations, angiographic patterns and outcomes of in-stent restenosis between bare-metal stents and drug-eluting stents. Eurolntervention 2010;5:841-6.

11 Dangas GD, Claessen BE, Mehran R, et al. Clinical outcomes following stent thrombosis occurring in-hospital versus out-of-hospital: Results from the HORIZONS-AMI (harmonizing outcomes with revascularization and stents in acute myocardial infarction) trial. J Am Coll Cardiol 2012;59:1752-9.

12 Habara S, Mitsudo K, Kadota K, et al. Effectiveness of paclitaxeleluting balloon catheter in patients with sirolimus-eluting stent restenosis. JACC Cardiovasc Interv 2011;4:149-54.

13 Scheller B, Hehrlein C, Bocksch W, et al. Treatment of coronary in-stent restenosis with a paclitaxel-coated balloon catheter. N Engl / Med 2006;355:2113-24.

14 Unverdorben M, Vallbracht C, Cremers B, et al. Paclitaxel-coated balloon catheter versus paclitaxel-coated stent for the treatment of coronary in-stent restenosis. Circulation 2009;119:2986-94.

15 Dangas GD, Claessen BE, Caixeta A, Sanidas EA, Mintz GS, Mehran R. In-stent restenosis in the drug-eluting stent era. J Am Coll Cardiol 2010;56:1897-907.

16 Lu G, Ades AE. Combination of direct and indirect evidence in mixed treatment comparisons. Stat Med 2004;23:3105-24.

17 Salanti G, Higgins JP, Ades AE, Ioannidis JP. Evaluation of networks of randomised trials. Stat Methods Med Res 2008;17:279-301.

18 Moher D, Liberati A, Tetzlaff J, Altman DG, Group P. Preferred reporting items for systematic reviews and meta-analyses: the PRISMA statement. BMJ 2009;339:b2535.

19 Hutton B, Salanti G, Caldwell DM, et al. The PRISMA extension statement for reporting of systematic reviews incorporating network meta-analyses of health care interventions: Checklist and explanations. Ann Intern Med 2015;162:777-84.

20 Higgins IPT, Green S. Cochrane handbook for systematic reviews of interventions 5.1.0 [updated March 2011]. www.cochranehandbook.org.

21 Mehran R, Dangas G, Abizaid AS, et al. Angiographic patterns of in-stent restenosis: Classification and implications for long-term outcome. Circulation 1999;100:1872-8.

22 Cutlip DE, Windecker S, Mehran R, et al. Academic Research Consortium. Clinical end points in coronary stent trials: a case for standardized definitions. Circulation 2007;115:2344-51.

23 Mauri L, Orav EJ, O'Malley AJ, et al. Relationship of late loss in lumen diameter to coronary restenosis in sirolimus-eluting stents. Circulation 2005;111:321-7.

24 Mauri L, Orav EJ, Candia SC, Cutlip DE, Kuntz RE. Robustness of late lumen loss in discriminating drug-eluting stents across variable observational and randomized trials. Circulation 2005;112:2833-9.

25 Lu G, Ades AE. Assessing evidence inconsistency in mixed treatment comparisons. I Am Stat Assoc 2006;447-59.

26 Dias S, Welton NJ, Caldwell DM, Ades AE. Checking consistency in mixed treatment comparison meta-analysis. Stat Med 2010;29:932-44 
27 Biondi-Zoccai G, Abbate A, Benedetto U, Palmerini T, D’Ascenzo F, Frati G. Network meta-analysis for evidence synthesis: what is it and why is it posed to dominate cardiovascular decision making? Int / Cardiol 2015;182:309-14

28 Dias S, Sutton AJ, Ades AE, Welton NJ. Evidence synthesis for decision making 2: A generalized linear modeling framework for pairwise and network meta-analysis of randomized controlled trials. Med Decis Making 2013;33:607-17.

29 Dias S, Welton NJ, Sutton AJ, Caldwell DM, Lu G, Ades AE. Evidence synthesis for decision making 4: Inconsistency in networks of evidence based on randomized controlled trials. Med Decis Making 2013;33:641-56.

30 Mills EJ, Thorlund K, loannidis JP. Demystifying trial networks and network meta-analysis. BMJ 2013;346:f2914.

31 Lambert PC, Sutton AJ, Burton PR, Abrams KR, Jones DR. How vague is vague? A simulation study of the impact of the use of vague prior distributions in MCMC using WinBUGS. Stat Med 2005;24:2401-28.

32 Brooks SP, Gelman A. General methods for monitoring convergence of iterative simulations / Comput Graph Stat 1998:7:434-55.

33 Salanti G, Ades A, loannidis JP. Graphical methods and numerical summaries for presenting results from multiple-treatment metaanalysis: an overview and tutorial. / Clin Epidemiol 2011:64:163-71.

34 DerSimonian R, Laird N. Meta-analysis in clinical trials. Control Clin Trials 1986;7:177-88

35 Higgins JP, Thompson SG, Deeks JJ, Altman DG. Measuring inconsistency in meta-analyses. BMJ 2003:327:557-60.

36 Borenstein M, Hedges LV, Higgins JPT, Rothstein HR. Introduction to meta-analysis. John Wiley \& Sons, 2009.

37 Chaimani A, Higgins JP, Mavridis D, Spyridonos P, Salanti G. Graphical tools for network meta-analysis in stata. PloS One 2013;8:e76654.

38 Peters JL, Sutton AJ, Jones DR, Abrams KR, Rushton L. Contourenhanced meta-analysis funnel plots help distinguish publication bias from other causes of asymmetry. / Clin Epidemiol 2008:61:991-6.

39 Peters JL, Sutton AJ, Jones DR, Abrams KR, Rushton L. Comparison of two methods to detect publication bias in meta-analysis. JAMA 2006; 295:676-80.

40 Egger M, Davey Smith G, Schneider M, Minder C. Bias in meta-analysis detected by a simple, graphical test. BMJ 1997;315:629-34

41 Lee JM, Park J, Kang J, et al. Comparison among drug-eluting balloon, drug-eluting stent, and plain balloon angioplasty for the treatment of in-stent restenosis: A network meta-analysis of 11 randomised, controlled trials. JACC Cardiovascular Intv 2015;8:382-94.

42 Hoffmann R, Mintz GS, Dussaillant GR, et al. Patterns and mechanisms of in-stent restenosis. A serial intravascular ultrasound study. Circulation 1996;94:1247-54

43 Shiran A, Mintz GS, Waksman R, et al. Early lumen loss after treatment of in-stent restenosis: An intravascular ultrasound study. Circulation 1998;98:200-3.

44 Park SJ, Kang SJ, Virmani R, Nakano M, Ueda Y. In-stent neoatherosclerosis: A final common pathway of late stent failure. J Am Coll Cardiol 2012:59:2051-7.

45 Alfonso F, Sandoval J, Perez-Vizcayno MJ, et al. Mechanisms of balloon angioplasty and repeat stenting in patients with drug-eluting in-stent restenosis. Int / Cardiol 2015:178:213-20.
46 Elezi S, Kastrati A, Neumann FJ, Hadamitzky M, Dirschinger J, Schomig A. Vessel size and long-term outcome after coronary stent placement. Circulation 1998:98:1875-80.

47 Kornowski R, Mintz GS, Kent KM, et al. Increased restenosis in diabetes mellitus after coronary interventions is due to exaggerated intimal hyperplasia. A serial intravascular ultrasound study. Circulation 1997;95:1366-9.

48 Cortese B, Berti S, Biondi-Zoccai G, et al. Drug-coated balloon treatment of coronary artery disease: A position paper of the Italian Society of Interventional Cardiology. Catheter Cardiovasc Interv 2014;83:427-35

49 Habara S, Kadota K, Kanazawa T, et al. Paclitaxel-coated balloon catheter compared with drug-eluting stent for drug-eluting stent restenosis in routine clinical practice. Eurolntervention 2015;10, doi:10.4244/EIJY15M02_09.

50 Kubo S, Kadota K, Otsuru S, et al. Everolimus-eluting stent implantation versus repeat paclitaxel-coated balloon angioplasty for recurrent in-stent restenosis lesion caused by paclitaxel-coated balloon failure. Eurolntervention 2015;10:e1-8

51 Windecker S, Kolh P, Alfonso F, et al. The task force on myocardial revascularization of the European Society of Cardiology (ESC) and the European Association for Cardio-Thoracic Surgery (EACTS) developed with the special contribution of the European Association of Percutaneous Cardiovascular Interventions (EAPCI). Guidelines on myocardial revascularization. Eur Heart J 2014;35:2541-619.

52 Mehran R, Dangas G, Abizaid A, et al. Treatment of focal in-stent restenosis with balloon angioplasty alone versus stenting short- and long-term results. Am Heart J 2001;141:610-4.

53 Alfonso F Zuecol, Cequier A et al. A randomised comparison of repeat stenting with balloon angioplasty in patients with in-stent restenosis. J Am Coll Cardiol 2003;42:796-805

54 Tomey MI, Kini AS, Sharma SK. Current status of rotational atherectomy. JACC Cardiovasc Intv 2014:7:345-53.

55 Albiero R, Silber S, Di Mario C, et al. Cutting balloon versus conventional balloon angioplasty for the treatment of in-stent restenosis: Results of the restenosis cutting balloon evaluation trial (RESCUT). J Am Coll Cardiol 2004:43:943-9.

56 Grasso C, Attizzani GF, Patanè M, Ohno Y, Capodanno D, Tamburino C. First-in-human description of everolimus-eluting bioabsorbable vascular scaffold implantation for the treatment of drug-eluting stent failure: Insights from optical coherence tomography. Int J Cardiol 2013;168:4490-1.

57 Alfonso F, Nuccio I, Cuevas C, Cardenas A, Gonzalo N, JimenezQuevedo P. Treatment of coronary in-stent restenosis with bioabsorbable vascular scaffolds. J Am Coll Cardio 2014;63:2875

58 Iabal J, Onuma Y, Ormiston J, Abizaid A, Waksman R, Serruys P. Bioresorbable scaffolds: Rationale, current status, challenges, and future. Eur Heart J 2014;35:765-76.

(C) BMJ Publishing Group Ltd 2015

Web appendix: Supplementary material 